\title{
Abl signaling shapes the intrinsic fluctuations of actin to direct growth of a pioneer axon in Drosophila
}

\author{
Akanni Clarke ${ }^{1,2}$, Philip G. McQueen ${ }^{3}$, Hsiao Yu Fang ${ }^{1}$, Ramakrishnan Kannan ${ }^{1}$, Victor Wang ${ }^{3}$, \\ Evan McCreedy ${ }^{3}$, Stephen Wincovitch ${ }^{4}$, and Edward Giniger ${ }^{1, *}$
}

$11{ }^{1}$ National Institute of Neurological Disorders and Stroke, National Institutes of Health, 12 Bethesda, MD 20892 USA

$14{ }^{2}$ The George Washington University School of Medicine/NIH Graduate Partnership Program, 15 Dept of Biochemistry and Molecular Medicine, Washington DC, 20037 USA

${ }^{3}$ Center for Information Technology, National Institutes of Health, Bethesda, MD 20892 USA

${ }^{4}$ National Human Genome Research Institute, National Institutes of Health, Bethesda, MD 20892 USA

* Corresponding author

25 Bldg 35, Rm 1C-1002

2635 Convent Dr.

27 Bethesda, MD 20892 USA

Tel: 1-301-451-3890

30 Fax: 1-301-451-5368

31 email: ginigere@ninds.nih.gov

32 ORCID: 0000-0002-8340-6158 


\section{Abstract}

34 The fundamental problem in axon growth and guidance is to understand how cytoplasmic signaling

35 modulates the cytoskeleton to produce directed growth cone motility. We show here that the TSM1

36 pioneer axon of Drosophila extends by using Abl tyrosine kinase to shape the intrinsic fluctuations

37 of a mass of accumulated actin in the distal axon. The actin mass fluctuates stochastically in length,

38 but with a small, forward bias that drives the axon along its trajectory by promoting emergence of

39 protrusions in leading intervals where actin accumulates, and collapse of protrusions in lagging

40 intervals that actin has vacated. The actin mass is sculpted by Abl signaling, which

41 probabilistically modulates its key parameters - its width and internal disorder - to drive its

42 advance, while maintaining internal coherence. Comparison of TSM1 to other systems suggests

43 that the mechanism we demonstrate here is apt to be common among pioneer axons in many

44 organisms. 


\section{Introduction}

The process of axon guidance is central to patterning the nervous system during development. Understanding how axons pathfind to their correct targets requires that we know the mechanism by which guidance information in the environment controls the spatial organization and dynamics of the growth cone cytoskeleton to produce directed extension of the axon. It is widely accepted that in some cases broad, flat, growth cones extend the axon by harnessing the mechanochemical properties of large adhesive lamellipodia ${ }^{1,2}$. There is reason to suspect, however, that other axons pathfind and extend in an entirely different way.

Cells in culture can switch mechanisms of cell motility, depending on the developmental context. For example, fibroblasts make large lamellipodia in 2-dimensional culture, yet assume spindle shaped morphologies and move faster in $3 \mathrm{D}$ environments ${ }^{3}$. Mesenchymal cells use a slow moving adhesive style of growth in $2 \mathrm{D}$ culture, but switch to a fast ameboid style of growth in $3 \mathrm{D}$ conditions of low adhesion and strong confinement ${ }^{4}$. Dendritic cells rely on integrins for 2D cell migration but 3D migration in vivo is protrusive and independent of integrin function ${ }^{5}$. These examples demonstrate that the molecular components and mechanisms used by diverse cell types to move in $2 \mathrm{D}$ versus $3 \mathrm{D}$ environments are consistently and fundamentally different.

Much as cell motility can be accomplished in multiple ways, there is also evidence for a second mode of axon growth. It has been reported that filopodial protrusions alone can extend some axons. In these cases, it seems that rather than applying adhesive traction to lamellipodia to pull the growth cone forward, axon growth is instead accomplished by selective stabilization and dilation of individual filopodia ${ }^{6,7}$. This filopodial style of growth has been proposed as a common mechanism for axon growth ${ }^{6,8}$, but it is not clear how such a mechanism would work, or how axon guidance signaling molecules would regulate it. 
To understand how signaling controls axon growth and guidance, there has been a

72 sustained effort to find and characterize individual molecules with the goal of linking them to

73 fundamental steps in the mechanism of axon extension and guidance. One such protein, the non-

74 receptor Abelson Tyrosine Kinase (Abl) acts downstream of nearly all of the common,

75 phylogenetically conserved families of axon guidance receptors (Bashaw et al., 2000; Crowner et

76 al., 2003; Forsthoefel et al., 2005; Wills et al., 1999 Dajas-Bailador et al., 2008; Yu et al., 2001).

77 Abl is critical for axon patterning (Grevengoed et al., 2001; Grevengoed et al., 2003; Liebel et al.,

78 2003; Koleske et al., 1998; Moresco et al., 2005 ${ }^{13,14}$, and acts in cooperation with a cohort of

79 accessory factors that regulate the organization and dynamics of the actin cytoskeleton, including

80 Enabled/VASP, Disabled, Abi, Trio, Rac and WAVE, among others ${ }^{15,16}$. We have recently

81 demonstrated that one key function of Abl signaling in axons is to balance linear extension of actin

82 filaments with actin branching, through its ability to suppress the activity of a linear actin

83 polymerizing factor (Enabled), and simultaneously stimulate an actin branching mechanism

84 (WAVE/Scar and Arp 2,3, under control of Trio and Rac) ${ }^{17}$. These roles place Abl at the key

85 interface between extracellular signaling and intracellular modulation of the axonal cytoskeleton,

86 though it remains unclear how these molecular interactions execute the cellular function of axon

87 growth and guidance.

We therefore set out to use live imaging of a single pioneer neuron in its native environment

89 to understand how Abl controls a pathfinding axon. We developed a method to image and

90 computationally quantify both the neuronal morphology and the cytoskeletal dynamics of the

91 TSM1 axon as it extends along its native trajectory in the intact Drosophila wing. We show here

92 that Abl regulates the organization and redistribution of an accumulated actin bolus that locally

93 controls filopodial morphogenesis to direct the extension and guidance of the TSM1 axon by 
94 locally regulating filopodial morphogenesis. Specifically, the distal portion of the TSM1 axon

95 accumulates a bolus of actin that displays forward-biased spatial fluctuations, leading over time to

96 its net advance during axon growth. Advance of the actin bolus, in turn, locally enhances the

97 density of filopodial protrusions in the region where actin moves to, and the disassembly of

98 protrusions in its wake, resulting over time in progressive advance of the filopodia-rich domain

99 that defines the growth cone morphologically. The redistribution of actin is itself coordinated by

100 the Abl tyrosine kinase signaling pathway, which modulates the spatial width of the actin

101 distribution and also minimizes its disorder, allowing predictable translocation of the growth cone.

102 Together, these data show how Abl probabilistically shapes the propagation of a leading actin mass

103 that directs the growth and guidance of a pioneer axon extending in its native environment.

104

105 Results

106 Abl regulates TSM1 axon patterning

In light of the historical difficulty in observing cytoskeletal dynamics in growing axons in

108 vivo ${ }^{19}$, we have developed a system amenable to such inquiry by employing a pioneer sensory

109 neuron of the Drosophila wing, called TSM1. During metamorphosis, the TSM1 axon extends in

110 the space between the dorsal and ventral epithelia of the wing, pioneering the growth of the L1

111 nerve laterally from the wing margin before turning and extending proximally toward the wing

112 hinge. The axon extends approximately $120 \mu \mathrm{m}$ over a 9 - $12 \mathrm{hr}$ period along this trajectory before

113 it fasciculates with the L3 nerve at the L1-L3 junction just distal to the GSR neuron (Figure 1A

114 and 1B) ${ }^{20}$. It has been shown previously that axons in cultured wing disc explants are robust and

115 pathfind faithfully ${ }^{20,21}$. Furthermore, the wing anatomy allows for an unobstructed view of TSM1

116 development. Therefore, TSM1 provides an excellent opportunity to observe the mechanism of

117 axonal growth and guidance in detail as the growth cone pathfinds through its native environment. 
We first verified that the Abl tyrosine kinase is required for growth and guidance of the

119 TSM1 axon, just as it is required for many other axons in the CNS and PNS of Drosophila, and of

120 vertebrates ${ }^{9-12}$. We found that perturbing Abl activity or expression caused aberrant TSM1 axon

121 growth and guidance phenotypes including instances where axonal growth stalled (Figure 1C), no

122 axon extended from the cell body (Figure 1D), the axon misrouted along its trajectory (Figure 1E),

123 and/or the axon maintained aberrant collateral branches (Figure 1C). Specifically, reducing Abl

124 expression by RNAi in the background of Dicer 2 overexpression (Abl KD) caused an increase in

125 aberrant TSM1 axon growth and guidance phenotypes (UAS-Abl RNAi, 51\% vs. control 11\%; p

$126<0.0001$, Figure 1F). The expressivity of TSM1 axon defects observed in Abl KD was enhanced

127 by heterozygosity for an Abl mutant allele (83\% with UAS-Abl RNAi/Abl4 vs. UAS-Abl RNAi,

$12850 \% ; p=0.002$, Figure $1 F)$, confirming the fidelity of the RNAi phenotype. Expression of Dicer2

129 alone also increased the frequency of subtle defects in TSM1 morphology (32\% vs 11\% in control,

130 Figure $1 \mathrm{~F}$ ) but the spectrum of defects is significantly different from that in the presence of Abl

131 RNAi ( $\mathrm{p}=0.0035$, chi-square, Supplemental Figure 2) and overexpression of Dicer 2 alone did

132 not affect cytoskeletal parameters measured in growing axons (see below). To discriminate the

133 catalytic function of Abl kinase from its scaffolding role, we treated wings with Imatinib, a specific

134 inhibitor of Abl Kinase activity, and found significant TSM1 axonal defects when compared to

135 control $(61 \%$ in $10 \mu \mathrm{M}$ Imatinib vs. control $11 \%$; $<0.0001$, Figure $1 \mathrm{~F})$. This result strongly

136 suggests that kinase activity is critical, though it does not exclude an important function for

137 scaffolding. We also observed axonal defects when Abl was overexpressed (Abl OE 35\% vs.

138 control 11\%; $\mathrm{p}=0.0123$, Figure $1 \mathrm{~F})$. Taken together, these results demonstrate that precise tuning

139 of Abl function is required for TSM1 axon extension and guidance. 


\section{Actin accumulates in the filopodial TSM1 growth cone}

We next live - imaged TSM1 axon extension in explanted early-pupal wing imaginal discs

143 by spinning disc confocal microscopy, with simultaneous visualization of the axonal membrane,

144 using neuron-specific expression of CD4 Tandem-Tomato, and intracellular actin, using

145 LifeactGFP (which labels both F- and G-actin ${ }^{22}$ ) in wild type, Abl KD and Abl OE backgrounds,

146 respectively. Multiplexed Z- stacks were collected every 3 minutes for 1.5 hours from fourteen

147 trajectories of each genotype. Axon morphology was traced stereoscopically in 3-dimensions, and

148 the intensity of the LifeactGFP signal was quantified along the axon shaft, from the base of the

149 axon to its distal tip. We focus on actin organization both because of its critical role in essentially

150 all forms of motility, and more specifically because the most extensively characterized output of

151 Abl signaling is its modulation of actin regulators. Control experiments showed that imaging of

152 developing transgenic wings in culture does not disturb the trajectory of the axon, or the ability of

153 these axons to reach the L1-L3 junction and fasciculate with the L3 nerve (data not shown).

Live-imaging revealed consistent, concerted advance of a zone of enhanced filopodial

155 density in the distal axon, together with an intra-axonal mass of accumulated actin, as the axon

156 extended. In all imaged timepoints, filopodia and transient axonal branches (referred to

157 collectively as protrusions) were the dominant morphological features of the axon. Large

158 lamellipodia were seen only very rarely ( $<1 \%$ of time points) (Figure $2 \mathrm{~A}-\mathrm{C})$. While the length

159 and density of protrusions were highly variable along the axon, a region with enhanced density of

160 protrusions was almost always observed in the distal portion of the axon (Figure 2B and E).

161 Similarly, we invariably observed a local accumulation of high actin intensity within the distal

162 axon (Figure 2B - D and Supplemental Figure 3), and this accumulation of actin advanced as the

163 axon grew (Figure 2G and 3E and Supplemental movie 1). We refer to the curve that reports the 
164 relative actin intensity as a function of its position along the axon as the "actin distribution"

165 throughout this manuscript (see, for example Fig 2D). Note that standard confocal imaging

166 methods can only record the dynamics of this bulk actin distribution, which incorporates actin

167 transport, diffusion, polymerization/de-polymerization, branching/de-branching, etc., it does not

168 resolve the motions of individual actin molecules. Actin accumulation in the distal axon was also

169 observed using other actin markers, including F-tractin Tandem-tomato, which selectively labels

170 F-actin, and actin-GFP, whereas a volume marker (cytoplasmic eGFP) showed little or no distal

171 accumulation (Supplemental Figure 4). Time-lapse image sequences suggested that the mass of

172 accumulated actin and the zone of enhanced protrusion density seemed to advance in concert as

173 the axon grew. To assess quantitatively the relationship between accumulated actin and protrusion

174 density along the axon, we first performed a sliding window analysis to determine the position of

175 the maximum value for each of these properties along the TSM1 axon in each image.

176 Quantification confirmed that the interval with the highest actin level (actin maximum) and the

177 interval with the highest protrusion density (protrusion maximum) were strongly correlated in

178 position along the axon, (Pearson $\mathrm{r}=0.71 ; \mathrm{p}<0.0001)$ (Figure $2 \mathrm{~F}$ ), and this held true across a

179 range of window sizes (range $1-10 \mu \mathrm{m}$ ). Furthermore, the correlation of actin intensity values

180 with local protrusion count was not restricted to the distal co-concentration of actin and

181 protrusions. Global comparison affirmed the relationship between actin intensity and local

182 protrusion count along the entire axon (Pearson $\mathrm{r}$ correlation $=0.60 ; \mathrm{p}<0.0001)$. This relationship

183 was also consistent when we compared the lengths of protrusions, rather than the count, with the

184 distribution of actin intensity (Pearson $\mathrm{r}$ correlation $=0.45 ; \mathrm{p}<0.0001$ ). These data demonstrate

185 that the length and number of axonal protrusions at particular positions along the axon shaft are

186 strongly correlated to the local amount of accumulated actin. 
Advance of the accumulated actin distribution predicts the local growth and disassembly of axonal protrusions

192 protrusion density are, in fact, slightly offset, with the actin maximum systematically leading the

193 maximum of the protrusion density as the axon grows (Figure 3). We aligned all 338-wildtype

194 time-points by the protrusion maximum along the axon and plotted the position of the actin 195 maximum, as well as the positions of the proximal and distal boundaries of the accumulated mass 196 of actin (referred to here as the "actin peak"). Empirically, we found it effective to define the 197 'edges' of the actin peak as the positions defined by the leading- and trailing- square root of the 198 second moment of the actin distribution about the actin peak (a measure akin to the standard 199 deviation of the distribution; see below and materials and methods) (Figure 3A). We found that 200 the actin peak enveloped the position of the maximum protrusion density: the leading 'edge' of 201 the actin peak leads the protrusion maximum by $6.7 \mu \mathrm{m} \pm 0.56(\mathrm{P}<0.0001)$, and the trailing 'edge'

202 follows the protrusion maximum by $8.5 \mu \mathrm{m} \pm 0.59(\mathrm{P}<0.0001)$, while the maximum of actin peak 203 led the maximum of the protrusion density by an offset distance of $2.8 \mu \mathrm{m} \pm 0.58$, (mean \pm SEM, $204 \mathrm{p}<0.0001$ ) (Figure 3B). As the TSM1 axon grows at an average rate of $0.23 \mu \mathrm{m} / \mathrm{min}$ (as measured 205 by a variety of metrics; see Supplemental Figure 5), this mean offset length implies that the 206 position of the actin peak predicts where the bulk of protrusions will be found 10-15 minutes later. 207 Furthermore, the offset distance between the actin maximum and that of the protrusion density 208 appears to be maintained actively. We found a strong negative correlation between the rate of 209 change of the offset distance between these two positions in pairs of successive time-points $(\mathrm{r}=-$ 
$2100.50, \mathrm{p}<0.0001$ ) (Figure 3C), which suggests that when the spacing between the actin and

211 protrusion maxima increases or decreases in any given time step, the gap size tends to be restored

212 during the next time point. Taken together, these data provide evidence that the actin accumulation

213 peak leads the advance of the axonal zone bearing the highest density of protrusions, with the

214 offset distance between these two axonal features being a regulated aspect of axon growth.

215 Based on these observations, we hypothesized that advance of the leading actin mass

216 increases the density of protrusions at the more distal position the actin peak now occupies, and

217 disassembly of protrusions at the position the actin has vacated. We therefore quantified the

218 protrusion density in intervals associated with the starting and ending positions of the actin peak

219 in 7 trajectories where the actin advanced $20 \mu \mathrm{m}$. Consistent with visual inspection of time lapse

220 micrographs of TSM1 axon extension dynamics, we found that, after a lag phase of $\sim 15$ min,

221 summed protrusion length declined by $50 \% \pm 0.1$ (mean \pm SEM, $\mathrm{p}<0.001)$ in the region vacated by

222 the actin peak, while at the new more distal position of the actin peak protrusion length was

223 increased by $32 \% \pm 0.1($ mean \pm SEM, $\mathrm{p}<0.01$ ), again with the actin peak on average reaching the

224 final position prior to the protrusion maximum (Figure 3D, Figure 3E, Supplemental Figure 6, and

225 Supplemental movie 1). Thus, the evolution of the actin distribution predicts where axonal

226 protrusions will subsequently grow and retract along the axon.

TSM1 axons maintain parameter relationships in Abl KD and OE genetic backgrounds

The observations above suggest that the molecular mechanism that controls the structure

230 and dynamics of the actin distribution is the key to regulating the morphological development and

231 extension of the TSM1 axon. We therefore imaged TSM1 axon extension in Abl KD and Abl OE

232 genetic backgrounds, computationally measured a broad set of parameters that capture axonal 
morphology and intra-axonal actin organization and compared these data sets to those from

234 wildtype TSM1 trajectories. Among the parameters we investigated were morphological features,

235 including the number, length, and branch order of protrusions, and the 3-dimensional volume of

236 substratum explored by protrusions from the growth cone (here called growth cone volume), as

237 well as measures of the actin distribution, including the length, organization, and speed of advance

238 of the peak of actin accumulation (see Table 1 and Materials and Methods for a complete listing

239 of imaged features and their definitions).

To verify that the mechanism underlying axon extension in the $\mathrm{Abl} \mathrm{KD}$ and $\mathrm{Abl} \mathrm{OE}$

conditions is fundamentally the same as that in wild type, we first queried the parameter set by

performing a Principal Components Analysis (PCA) using the wild type dataset of growth cone measurements (Supplemental Figure 7). The first two principal components account for $47 \%$ of

244 the total variance in the data set. PC1, which accounts for $29 \%$ of the variance, is dominated by

245 contributions from morphological parameters including the number and length of protrusions

246 along the axon, while PC2 (18\% of the variance) is dominated by parameters that measure how

247 the actin distribution is organized. We applied the two eigenvectors to the Abl KD and Abl OE

248 data sets to compare how similar the global relationships among parameters in the altered-Abl

249 conditions are to those in wild type (Figure 4A). A substantial number of data points from the Abl

250 perturbed conditions occupied the same PC1 vs. PC2 parameter space as did the wild type data set.

251 Crucially, however, the data points from Abl KD and Abl OE that did not overlap wild type did

252 not just spread out isotropically, nor did they segregate into a discrete, separate domain of

253 parameter space. Instead, they formed a restricted distribution along a single vector emanating

254 from the cloud of wild type data. Stated otherwise, the ratio of the values of any pair of principal

255 components in the Abl-perturbed conditions varied along a simple, linear relationship relative to 
their values in wild type, even for the data points with the most severely altered absolute values.

257 This pattern was observed in all pairwise comparisons among the first 4 principal components,

258 which account for $71 \%$ of the variance in the data set, thus revealing consistent quantitative

259 relationships among the growth cone parameters that produce morphology and motility in

260 wildtype, $\mathrm{Abl} \mathrm{KD}$ and $\mathrm{Abl} \mathrm{OE}$. This verifies that the fundamental relationships among

261 morphological and cytoskeletal properties of wild type growth cones are maintained in the Abl

$262 \mathrm{KD}$ and OE conditions, even for the most severely affected data points in our dataset, and therefore

263 validates the use of the altered Abl conditions to interrogate wild type growth cone dynamics.

Abl perturbation has large effects on the actin distribution and relatively smaller effects on

growth cone morphology

Comparison of parameters across all three genotypes revealed that the actin distribution was far more sensitive to changes in Abl levels than were the morphological parameters of the axon, consistent with the hypothesis that actin remodeling may be the more direct mechanistic target of Abl-dependent signaling, upstream of changes in axonal morphology. Abl perturbation

271 affects many growth cone parameters, but to varying degrees. For example, the mean length of the 272 actin peak is decreased in the knockdown condition, and increased by Abl OE, yet is unaffected 273 by expression of Dicer 2 alone (mean \pm SEM, $15.16 \mu \mathrm{m} \pm 0.19$ in control vs. $14.0 \mu \mathrm{m} \pm 0.27$ in $274 \mathrm{KD} ; \mathrm{p}=0.0051,15.83 \mu \mathrm{m} \pm 0.24$ in Dicer 2; N.S., and $16.72 \mu \mathrm{m} \pm 0.32$ in OE; $\mathrm{p}=0.0002$; One 275 way ANOVA) (Figure 4B). Similarly, the length of the protrusion peak in Abl KD is smaller than 276 wildtype $(15.9 \mu \mathrm{m} \pm 0.21$ in control vs. $14.5 \mu \mathrm{m} \pm 0.18$ in $\mathrm{KD} ; \mathrm{p}<0.05)$ and is larger in Abl OE

$277(18.9 \mu \mathrm{m} \pm 0.27 ; \mathrm{p}<0.001)($ Figure 4C). To determine which aspects of TSM1 architecture were 278 most affected by Abl, we calculated the Z-score for each parameter relative to the wildtype mean. 
While disruption of Abl signaling does not shift the mean value of any morphological feature by more than 0.7 standard deviations from its wildtype mean, actin parameter values are shifted by as much as $2-11$ standard deviations away from their respective control means (Figure 4D). As Abl perturbation causes substantially larger effects on the distribution of actin in the axon as compared to axonal morphology, we infer that the actin distribution is the more sensitive target of Abl 284 signaling.

Net forward motion of the actin distribution arises from biased stochastic fluctuations of the

distribution

In light of the observation that the shape of the actin distribution is a sensitive target of guidance signaling downstream of $\mathrm{Abl}$, and that the pattern of actin redistribution predicts where future axonal protrusions will grow, we next examined the evolution of the actin distribution itself over time in each trajectory. We found that anterograde translocation of the actin peak was not consistent. Rather, both the maximum of the actin peak, and its midpoint, displayed extensive, seemingly stochastic, fluctuations proximo-distally along the axon, but with a small anterograde

294 bias that resulted, over time, in net forward motion of the actin peak (Figure 5B). This bias in the 295 stochastic motion of these positions is evident upon tracking the positions of the front, rear and maximum of the actin distribution over time for any single trajectory (Figure 5A). Further

297 inspection of actin redistribution patterns revealed that the position of the actin maximum largely

298 fluctuates at each time step within the domain defined as the actin peak (i.e. the window defined

299 by the leading and trailing square root of the second moment of the actin peak; $77 \%$ of time steps

300 in wild type (251/327). Consistent with this, we found that a broader actin peak allowed larger

301 excursions of the maximum and midpoint, while narrow actin peaks are correlated with relatively 
short fluctuations of the position of the actin max (Pearson $r=.24, p<0.0001$ ) (Figure 5C). The net effect is that the actin distribution displays an 'inchworm' style of motion, with the front of the actin peak advancing over time, and the rear catching-up (Figure 2G).

\section{Actin accumulation is fragmented and advance of the distribution is unpredictable in Abl-}

\section{perturbed backgrounds}

The most striking consequence of Abl deregulation in individual images is fragmentation of the actin distribution (Figure 6A - D). We quantified this phenotype using a measure from information theory called the Fisher Information Content (FI), which measures the coherence of the actin distribution, including both short-range fluctuations of intensity $(<1 \mu \mathrm{m}$ spatial scale) and mid - long range fragmentation of actin $(2-20 \mu \mathrm{m}$ scale). We find that the actin distribution profiles from Abl perturbed axons are vastly fragmented as compared to controls (3.87 \pm 0.14 in control vs. $32.67 \pm 1.76$ in $\mathrm{Abl} \mathrm{KD}$ and $18.54 \pm 1.40$ in $\mathrm{Abl} \mathrm{OE}$; mean $\pm \mathrm{SEM}, \mathrm{p}<0.001$ in each comparison) This suggests that one effect of $\mathrm{Abl}$ is to regulate the coherence of the actin distribution in the axon.

Next, we compared the actin distribution dynamics in the wildtype condition with the Abl perturbed conditions and found that the actin distribution evolves in an orderly way from one-time step to the next in wild type, but much less so when $\mathrm{Abl}$ is dysregulated (Figure 6E - 6H', and Supplemental movie 2 - 4). The evolution of the actin distribution was quantified using another approach from information theory, called the Jensen Shannon Divergence (JSD), which calculates the dis-similarity ("divergence") of the shape of the actin distribution between pairs of time points in a given trajectory. Thus, two distributions that are very similar have very low divergence (JSD close to 0), while two distributions that are very different have high divergence (JSD close to 1). 
In the control condition, successive time points of any single trajectory tended to have highly overlapped distributions, and thus low JSD values, and the overlap tended to decay in an orderly way as comparisons were made to more distant time points. In contrast, in the Abl KD and Abl

328 OE conditions, the overlap between nearest neighbor time points was commonly as dissimilar as two distributions separated by multiple time steps in the wildtype condition, as indicated by increased mean JSD values $(0.17 \pm 0.0039$ in the control vs. $0.38 \pm 0.011$ in the KD and $0.29 \pm$ 0.011 in the $\mathrm{OE}$; mean \pm SEM $\mathrm{p}<0.001)$. These data demonstrate dynamic order in the temporal evolution of the actin distribution in each WT TSM1 trajectory, while in the Abl perturbed conditions, that same evolution is dynamically disordered and unpredictable.

\section{Actin disorganization correlates with morphological phenotypes in Abl perturbed}

\section{backgrounds}

While the strongest effects of Abl perturbation on single parameters were on those measuring aspects of the actin distribution, analysis of parameter interactions suggested that the critical function of $\mathrm{Abl}$ lies in the linkage of those actin parameters to their morphological

340 consequences. This was revealed most clearly by the morphological correlates of the extremes of

341 those actin values. Thus, for example, high fragmentation of the actin distribution was

342 preferentially associated with the smallest growth cones in $\mathrm{Abl} \mathrm{KD}(\mathrm{r}=-0.14 ; \mathrm{p}=0.005)$, but it

343 was associated with the largest GCs in Abl OE $(r=0.25 ; \mathrm{p}<0.0001)$ (Figure 6I). In the case of

$344 \mathrm{Abl} \mathrm{KD}$, this reflected a population of very short growth cones with hypercondensed actin, that is,

345 growth cones containing small, intensely-labelled foci of actin. Data presented above showed that

346 actin step size, ie the distance the actin maximum (or midpoint) advances in any single time step,

347 is correlated with the length of the actin peak (Figure 5C). In Abl KD, however, the growth cone 
often contracts beyond the lower limit observed in wild type, and the step size falls to nearly zero

349 (Figure 6J). This is a genotype in which we observe growth cone stalling, and failure to form an axon. In contrast, in $\mathrm{Abl} \mathrm{OE,} \mathrm{a} \mathrm{genotype} \mathrm{associated} \mathrm{primarily} \mathrm{with} \mathrm{axon} \mathrm{misrouting,} \mathrm{maximal} \mathrm{actin}$

351 fragmentation is associated preferentially with the largest actin distributions, and in particular, with

352 distributions broader than the upper limit typically observed in wild type (Figure 6K). These

353 abnormally broad distributions often greatly exceed the distance over which the motions of

354 individual actin molecules display correlation within a disordered actin network (decay length

$355 \sim 11-16 \mu \mathrm{m}$; Ref 23). The potential molecular basis of these length-dependent changes in actin 356 organization, and the consequences they are predicted to have for orderly axon growth, will be 357 considered in detail in the Discussion.

\section{Discussion}

What is a growth cone, and how does it extend an axon? We have shown here that a local

361 accumulation of actin in the distal axon generates the zone of enhanced protrusive dynamics that

362 defines the TSM1 growth cone. Over time, this actin bolus advances down the nascent axon,

363 supporting formation of new filopodial protrusions from leading intervals now bearing enhanced

364 actin levels, thus enabling extension of the axon. Protrusions left behind in the wake of actin

365 advance are fated for disassembly, thus consolidating the proximal axon. Inchworm-like dynamics

366 advance the actin mass with an anterograde bias that moves the distribution forward over time.

367 Furthermore, our data demonstrate that the Abelson tyrosine kinase, a conserved regulator of 368 cytoskeletal dynamics that signals downstream of many guidance cue receptors, coordinates the 369 actin fluctuations that sum to produce the net forward motion of the distribution. Taken together, 370 our data suggest that the fundamental function of Abl during axon guidance and extension is to 
371 modulate, in a probabilistic way, the fluctuations and the coherence of an advancing actin wave

372 that directs the construction and consolidation of the growing axon in response to guidance cues

373 (Model Figure 7).

374 Growth cone advance is often discussed by invoking deterministic, clutch-like adhesive 375 mechanisms that harness the mechanochemical properties of leading lamellipodia ${ }^{1,2,24}$. For some 376 neurons, particularly those extending axons on relatively rigid, highly adherent substrata, these 377 models provide a plausible explanation for axon growth. However, TSM1 and many other axons 378 look non-lamellar, particularly in compliant, 3D environments like those often encountered by 379 pioneer axons in vivo ${ }^{6,7,25,26}$. In these contexts, growth cones are often dominated by filopodial 380 protrusions and seem to lack the large veil-like structures that we associate with the adhesive style 381 of growth. Moreover, the growth of these axons appears to be accomplished by protrusion and 382 selective stabilization of filopodia, rather than by traction forces applied to the lamellipodia-like 383 veils between filopodia (O'Connor et al., 1990; Sabry et al., 1991). These contrasting styles of 384 axon growth are reminiscent of the dichotomy between cellular motility on rigid 2D substrata vs 385 protrusive cell motility in compliant 3D environments. They also add support to the growing notion 386 that the mechanisms of motility employed by neurons are not fixed, but are instead dependent on 387 the context of their environment. We now find that the filopodial-dominated TSM1 growth cone extends its axon within the 389 intact Drosophila wing by regulated advance of an actin distribution, using a protrusive 390 mechanism of motility that is probabilistic rather than deterministic, and is based on the statistical 391 properties of disordered actin networks. Forward motion of the actin peak arises from a small 392 spatial bias that is applied to the fluctuating actin distribution; forward motion of the axon terminus 393 arises from preferential extension of filopodia from axon regions that now have high actin density 
394 and retraction of filopodia in regions of low actin density. In essence, the advancing actin peak

395 directs processive axon growth by locally promoting assembly of potential axonal tracks, while

396 the axon cannibalizes filopodia that lag behind the actin peak, and correspond to axonal paths that

397 were not taken.

The key to such a mechanism for axon extension is that forward expansion of the actin

399 distribution must be large enough to advance the actin bolus and produce net growth, yet

400 constrained enough that the actin bolus remains coherent and the growth cone behaves as a single,

401 unitary entity. We see here that balancing these two competing, yet related, requirements is the

402 fundamental role of Abl tyrosine kinase signaling. It is well established that signaling by the Abl

403 network must be maintained at an intermediate level of activity to support proper growth and

404 guidance of axons (Grevengoed et al., 2001; Grevengoed et al., 2003; Liebel et al., 2003; Koleske

405 et al., 1998; Moresco et al., 2005). Our data suggest that this intermediate level is crucial for two

406 reasons. First, it minimizes the disorder of the accumulated actin distribution, as assayed both

407 instantaneously, by the Fisher Information of the actin profile at each time step, and also

408 dynamically, by the Jensen-Shannon analysis of the evolution of the distribution between time

409 steps. Second, we also found that Abl regulates the width of the actin peak. This is seen most

410 clearly in the Abl perturbations, where reducing Abl promotes condensation of the actin mass

411 while increasing Abl promotes expansion of the peak. Specifically, Abl knockdown causes the

412 actin bolus to hyper-condense and fragment into small, tightly-packed foci that show reduced

413 spatial motion (Figure 4 and 6). Since expansion of the distribution provides the forward motion

414 required for 'inchworming', it is plausible that failure of that expansion in the Abl KD condition

415 is responsible for the growth cone stalling observed in this genotype. Conversely, overexpression

416 of Abl causes the actin distribution to expand, often to the degree that the peak exceeds the 
417 characteristic decay length over which the motions of individual actin molecules remain correlated

418 within disordered actin networks $(\sim 11-16 \mu \mathrm{m}){ }^{23}$. This likely contributes to the preferential

419 fragmentation we observe of the broadest actin peaks in the Abl OE condition, and plausibly could

420 predispose to axonal misrouting and inappropriate axon branching in part by allowing different

421 portions of the same growth cone to act independently. The two key functions of Abl, minimizing

422 disorder and controlling distribution width, are closely intertwined; in the Abl KD condition the

423 tendency is for the smallest growth cones to drive measures of disorder, while in Abl OE, the

424 degree of actin disorder increases with expanding length of the actin peak (Fig 6I).

Our model of growth cone behavior is derived partly from the consequences of vigorous

experimental perturbations of Abl, but our goal is to understand the consequences of modest

manipulated experimental contexts are a faithful mirror of the relationships among those

433 albeit to a more measured extent. Hence, we would predict, for example, that a guidance cue that 434 enhances Abl activity, such as Netrin acting through DCC/Frazzled ${ }^{27,28}$ would promote expansion

435 of the actin domain, and thus bias growth, in the direction of greater cue concentration, while local 436 accumulation of a cue that leads to reduced Abl activity, such as Delta acting through the receptor 437 Notch 10,29,30 would locally promote condensation of the actin, and therefore focus future 438 proliferation of filopodia from the vicinity of that accumulation. Similarly, by this model it is 439 straightforward to see why the subcellular localization that has been observed for some guidance 
receptors within specific subregions of the growth cone (V. Castellani, pers comm, Sept 2018)

441 might be essential for them to promote either axon extension or retraction, respectively, or how a

442 single receptor could be switched between attraction and repulsion simply by changing the polarity

443 of its distribution in the growth cone.

A crucial mechanistic question raised by our experiments is how varying the level of Abl

445 activity produces changes in the width of the actin peak. We have shown recently that the structure

446 of the Abl signaling network intrinsically causes it to modulate the ratio of linear vs branched actin

447 within the cell ${ }^{17}$. Thus, activating Abl suppresses Enabled, a factor that extends linear actin

448 polymers, but stimulates the Trio, Rac1, WAVE/SCAR, Arp2,3 axis that leads to actin branching.

449 Suppressing Abl activity has the opposite pair of effects. Remarkably, a number of recent

450 experimental and computational analyses have demonstrated that simply altering the ratio of linear

451 vs branched actin in a disordered network is sufficient to alter network dimensions in just the way

452 we observe in TSM1 ${ }^{23,31,32}$. Thus, manipulations that produce increased network crosslinking, such

453 as a higher proportion of branched actin, tend to expand a moderately cross-linked actin network,

454 while increasing the proportion of unbranched, linear actin favors coalescence of the distribution.

455 These consequences of actin network modulation are consistent with data presented here which

456 show that the Abl KD condition causes growth cones with short, hypercondensed actin

457 distributions to be overrepresented, while in the Abl OE condition, growth cones with longer,

458 extended actin distributions are overrepresented (Figure 4 and Supplemental Figure 8). Therefore,

459 it is possible that the effect of $\mathrm{Abl}$ on the actin distribution, and therefore on morphological growth

460 and guidance, could be accounted for simply by the demonstrated biochemical effects of Abl on

461 pathways leading to branched vs linear actin. This conjecture will be investigated experimentally

462 in future studies. 
It is well-established that $\mathrm{Abl}$ has other roles aside from regulating actin extension and

464 branching, in particular regulation of the microtubule plus-end tracking protein Orbit ${ }^{33}$. Our data

465 do not exclude the idea, for example, that Abl-dependent interaction of actin with microtubules

466 could contribute to the forward-bias of actin fluctuations, perhaps by providing directionality.

467 Additionally, the Abl network may also influence axon extension and guidance through more

468 direct effects on morphological features of the growth cone ${ }^{27,28}$. Finally, in this analysis, we have

469 tracked the bulk distribution of actin. The motions of individual actin molecules remain unknown.

470 Evolution of the actin distribution presumably incorporates actin polymerization,

471 depolymerization, branching, crosslinking, diffusion, myosin contractility, active transport via

472 microtubules, and bulk flow of actin from the cell body, among other contributors. Future

473 experiments will aim to separate the individual contributions of these processes.

$474 \quad$ The mechanism of axon growth we observe here is energetically expensive, with only $\sim 5-$

$47515 \%$ of the total back-and-forth motion of the actin peak being captured in net advance of the actin

476 mass. Considered in context, however, this mechanism allows an extremely efficient use of

477 guidance information. The fluctuations of the actin peak cause it to repetitively sample leading and

478 trailing positions along the axon multiple times before moving irrevocably along its trajectory. We

479 propose that this is useful, and probably essential, for responding accurately to the shallow, often

480 noisy gradients of individual guidance cues, which are typically presented in complex

481 combinations in the substratum. In this sense, the mechanism we have found is most akin to the

482 mechanism of bacterial chemotaxis, in which external attractants and repellants provide only a

483 subtle spatial bias to essentially stochastic fluctuations of the motility machinery, relying on a

484 'random walk with a ratchet' to produce net guidance ${ }^{34,35}$. Additionally, we note that these actin

485 fluctuations survey all growth cone protrusions - off-axis lateral projections as well as on-axis 
extensions of the axon shaft- thereby allowing growth cone turning to derive from precisely the same machinery as does linear extension of the axon. Abelson tyrosine kinase, which probabilistically localizes the site of filopodial dynamics, represents a novel mechanism of motility that drives the extension and guidance of the TSM1 axon

491 in the intact Drosophila wing. This style of growth expands our understanding of the fundamental mechanisms used by neurons to build neural circuits and reveals a novel molecular and cellular mechanism for $\mathrm{Abl}$, one of the central regulators of cell morphology and neural wiring.

Materials and Methods

Drosophila stocks

Neuralized-Gal4, Abl knock down (KD) (UAS-Abl-RNAi), Abl overexpression (OE) (UAS-

$A b l$ ), UAS Dicer2, and the $a b l^{4}$ allele have been described previously ${ }^{17,18}$. UAS-lifeactGFP, and

UAS-CD4+ tandem tomato stocks were obtained from the Bloomington Drosophila stock center.

\section{TSM1 axon growth and guidance phenotypes}

White pre-pupae marked at the start of pupariation in each genotype were aged $7.5-8 \mathrm{hrs}$

503 at $25^{\circ} \mathrm{C}$. Aged pupae were dissected in fresh culture media (CM) composed of Schneider's

504 Drosophila media (Life Technologies) supplemented with 10\% fetal bovine serum (Gibco).

505 Wings were removed and placed in a 35mm MatTek glass bottom microwell petri dish ventral side

506 down and a halved circular cover slip was placed on their dorsal surface. Discs were slightly

507 compressed to immobilize and flatten the tissue. Inert, compressible clay was placed between the

508 bottom interior surface of the MatTek dish and the top cover glass to prevent over compression of 
509 the wings and to adhere the top coverslip to the bottom of the culture chamber (Supplemental

510 Figure 1). $5 \mathrm{ml}$ of $\mathrm{CM}$ was added to the dish after the tissue was secured in position. To assay

511 terminal axonal phenotypes, wing discs were aged for $\sim 16$ hours at $25^{\circ} \mathrm{C}$ and microscopy was

512 performed on a Zeiss NLO 880 w/Airy scan confocal microscope using a 25X 1.4 NA water

513 immersion objective. Non-wildtype TSM1 axons assumed one of four distinguishable phenotypes:

514 no axon extended, axonal stall, guidance defect, or aberrant collateral branches. For simplicity, all

515 non-wildtype phenotypes were scored as aberrant.

\section{Pharmacological inhibition of Abl}

A 100mg tablet of Imatinib (Novartis, obtained from the NIH Clinical Center Pharmacy)

was dissolved in $20 \mathrm{ml}$ of Schneider's Drosophila medium producing a 10mM stock, and aliquots were stored at -20C. 5 ul of 10mM Imatinib/Schneider's suspension was added to $4.995 \mathrm{ml}$ of CM

521 for a final experimental concentration of $10 \mu \mathrm{M}$. $5 \mathrm{ml}$ of Fresh $10 \mu \mathrm{M}$ Imatinib $\mathrm{CM}$ was added to 522 wildtype wing discs mounted as described above. Cultures were aged and imaged as described 523 above.

\section{Growth cone time-lapse imaging}

Wing discs for the control, Abl-KD and Abl-OE conditions were prepared and mounted as

527 described above for scoring aberrant end-stage phenotypes. Live-imaging was performed

528 immediately after mounting at $25^{\circ} \mathrm{C}$ using an inverted Zeiss Axio Observer Z1 confocal

529 microscope fitted with a Yokogawa Spinning Disk (SD) module and a temperature-controlled

530 stage. Z-stacks at 0.8 um spacing were collected from individual TSM1 axons every 3 minutes 
531 using a $63 \mathrm{X} 1.2 \mathrm{NA}$ water immersion objective. (Note that laser power was set at a minimal value

532 to mitigate photodamage and ensure image intensities were not saturated.)

534 Segmentation of the axon and quantification of growth cone parameters

Neuronal z stacks were stereoscopically reconstructed in 4-dimensions (x, y, z, and time) using IMARIS (Bitplane, version 8.0.2) and segmented using the semi-automatic 'filament tracer'

537 function. The axonal backbone and protrusions of TSM1 were identified and traced using the 538 membrane localized CD4+ tandem tomato signal; false positives were removed manually, and untraced protrusions were added manually. Tracings were exported as inventor files (.iv) and converted to the standard SWC file format using a MiPav plugin. Morphological parameters

541 extracted from these segmented images included the number of protrusions, length of each

542 protrusion, position of individual protrusions along the axon, and protrusion branch order among 543 others (for a full list of measured parameters, see Table 1)

544 Custom scripts in Mathematica software were written to identify computationally the 545 position of highest filopodial density along the axon by using a sliding-window method to sum the 546 length and number of protrusions within a $5 \mu \mathrm{m}$ window that advanced $1 \mathrm{um} /$ per step along the 547 segmented axon. We varied window size from 1-10 $\mu \mathrm{ms}$ and empirically found peak protrusion 548 density assignment was insensitive to the size of the window (data not shown). We then calculated 549 the square root of the second moment about the peak of protrusion density to determine the length 550 of the protrusive zone, both separately for the portions of the distribution leading and trailing the 551 peak position, and also globally to measure the length of the entire distribution. 
SWC files from a complete trajectory of TSM1 growth and the corresponding 4D Z stacks,

555 (x, y, z, time) converted to Nikon image cytometry standard (ICS) format in Imaris, were loaded

556 into two MiPav plugins, PluginDrosophilaCreatesSWC and PlugIn3DSWCStats to extract the

557 actin distributions from each time point. Plugin code is available in MiPav, but its function is

558 described below.

559 In brief, image intensity is calculated by summing the actin intensity within sequential

560 frustums that encompass the axon. First, the MiPav plugin performs a background subtraction on

561 each image. A 3D probability map of intensity values is then generated to determine the boundaries

562 of the actin signal. A circle is then expanded from each coordinate in the SWC tracing to determine

563 the radius of each frustum. The radius is normal to the SWC axis, and is bounded by the

564 background of the image in the probability map. Summed intensity values from non-overlapping

565 frustums are then reported as a function of position along the axonal tracing. Actin peak position

566 was identified by sliding window and the square root of the second moment of the distribution was

567 calculated just as for the equivalent measurements of filopodial density.

569 Fisher Information

Fragmentation of the actin distribution was quantified by the Fisher Information (FI),

571 which is a measure of the amount of information that can be specified by the shape of a distribution.

572 The FI for each actin distribution profile was calculated according to the formula:

$$
\int d x\left(\frac{d\left(p(x)^{1 / 2}\right)}{d x}\right)^{2}
$$

574 using the trapezoid approximation to the integral, where $\mathrm{p}(\mathrm{x})$ is the distribution of actin intensity

575 (x) values for each actin profile. In this context, FI yields a quantity representative of the 576 fragmentation of the actin distribution. 
Jensen Shannon Divergence

The Jensen-Shannon divergence (JSD) was used to quantify the divergence, or overlap,

between actin distributions at different time steps of any single trajectory according to the formula:

$$
J S D(p \| r)=\frac{1}{2} \sum_{n=1}^{n=N}\left[p_{n} \log _{2}\left(\frac{2 p_{n}}{p_{n}+r_{n}}\right)+r_{n} \log _{2}\left(\frac{2 r_{n}}{p_{n}+r_{n}}\right)\right]
$$

where $\mathrm{p}$ and $\mathrm{r}$ are two probability distributions, i.e. actin distributions, Since the JSD does

not satisfy the triangle inequality, for the principal component analysis in this report we used the

square root of the JSD, the Jensen -Shannon metric (JSM) which can be used as a metric.

\section{Quantification of protrusions in response to actin distribution translocation}

To quantify the effect that actin advance has on local axonal protrusion, we summed the (MATLAB).

\section{Statistics and reproducibility}

594 are reported in text and figure legends above.

\section{Data and Code availability}

597 Numerical data for all figures is included in Supplemental datasheet 1. MiPav plugin code for 
599 NIH image analysis package package, MiPav. Matlab and Mathematica scripts will be made

600 available upon publication by uploading to the publicly accessible NIH website:

601 https://data.ninds.nih.gov

602

603 Acknowledgements

604

605

We wish to thank all the members of our lab for their advice and assistance during the course of

606 these experiments, particularly Kate O’Neill for Matlab expertise. We would also particularly like

607 to thank Chi-Hon Lee, Sally Moody, Clare Waterman, Bob Fischer, Chun-Yuan Ting, Lenny

608 Campanello, Wolfgang Losert and Garyk Papoian for their many helpful suggestions, and Laura

609 Alto, Jon Terman and Ken Yamada for comments on the manuscript. Additionally, we thank Mike

610 Murrell and Ian Linsmeier for sharing their data and ideas about the motions of actin molecules,

611 and Valerie Castellani for sharing unpublished data on fine-scale localization of guidance receptors

612 in the growth cone. Many Drosophila stocks were provided by the Bloomington Drosophila Stock

613 Center. These experiments were supported in part by the Basic Neuroscience Program of the

614 NINDS Intramural Research Program (Z01-NS003013 to EG). PGM, VW and EMcC were

615 supported by the Intramural Research Program of NIH, CIT, and SW was supported by the

616 NHGRI, NIH. RK was supported in part by a DBT Ramalingaswami re-entry fellowship from the

617 Government of India.

618

\section{Competing interests}

620 The authors declare no competing interests.

References 
623 1. Lewis, A. K. \& Bridgman, P. C. Nerve growth cone lamellipodia contain two populations of

624 actin filaments that differ in organization and polarity. J. Cell Biol. 119, 1219-1243 (1992).

625 2. Lin, C.-H. \& Forscher, P. Growth cone advance is inversely proportional to retrograde F-

626 actin flow. Neuron 14, 763-771 (1995).

627 3. Cukierman, E., Pankov, R., Stevens, D. R. \& Yamada, K. M. Taking Cell-Matrix Adhesions

628 to the Third Dimension. Science 294, 1708-1712 (2001).

629 4. Liu, Y.-J. et al. Confinement and Low Adhesion Induce Fast Amoeboid Migration of Slow

$630 \quad$ Mesenchymal Cells. Cell 160, 659-672 (2015).

631 5. Lämmermann, T. et al. Rapid leukocyte migration by integrin-independent flowing and

632 squeezing. Nature 453, 51-55 (2008).

633 6. O’Connor, T. P., Duerr, J. S. \& Bentley, D. Pioneer growth cone steering decisions mediated 634 by single filopodial contacts in situ. J. Neurosci. 10, 3935-3946 (1990).

635 7. Sabry, J. H. et al. Microtubule behavior during guidance of pioneer neuron growth cones in 636 situ. J. Cell Biol. 115, 381-395 (1991).

637 8. Grabham, P. W., Reznik, B. \& Goldberg, D. J. Microtubule and Rac 1-dependent F-actin in 638 growth cones. J. Cell Sci. 116, 3739-3748 (2003).

639 9. Bashaw, G. J., Kidd, T., Murray, D., Pawson, T. \& Goodman, C. S. Repulsive Axon

640 Guidance: Abelson and Enabled Play Opposing Roles Downstream of the Roundabout

$641 \quad$ Receptor. Cell 101, 703-715 (2000).

642 10. Crowner, D., Le Gall, M., Gates, M. A. \& Giniger, E. Notch Steers Drosophila ISNb Motor

643 Axons by Regulating the Abl Signaling Pathway. Curr. Biol. 13, 967-972 (2003). 
644 11. Forsthoefel, D. J., Liebl, E. C., Kolodziej, P. A. \& Seeger, M. A. The Abelson tyrosine

645 kinase, the Trio GEF and Enabled interact with the Netrin receptor Frazzled in Drosophila.

646 Development 132, 1983-1994 (2005).

647 12. Wills, Z., Bateman, J., Korey, C. A., Comer, A. \& Van Vactor, D. The Tyrosine Kinase Abl

648 and Its Substrate Enabled Collaborate with the Receptor Phosphatase Dlar to Control Motor

649 Axon Guidance. Neuron 22, 301-312 (1999).

650 13. Dajas-Bailador, F., Jones, E. V. \& Whitmarsh, A. J. The JIP1 Scaffold Protein Regulates

651 Axonal Development in Cortical Neurons. Curr. Biol. 18, 221-226 (2008).

652 14. Yu, H.-H., Zisch, A. H., Dodelet, V. C. \& Pasquale, E. B. Multiple signaling interactions of

653 Abl and Arg kinases with the EphB2 receptor. Oncogene 20, 3995-4006 (2001).

654 15. Grevengoed, E. E., Fox, D. T., Gates, J. \& Peifer, M. Balancing different types of actin

655 polymerization at distinct sites: roles for Abelson kinase and Enabled. J. Cell Biol. 163,

$656 \quad 1267-1279(2003)$.

657 16. Song, J. K. et al. Disabled is a bona fide component of the Abl signaling network.

$658 \quad$ Development 137, 3719-3727 (2010).

659 17. Kannan, R. et al. The Abl pathway bifurcates to balance Enabled and Rac signaling in axon

660 patterning in Drosophila. Development 144, 487-498 (2017).

661 18. Kannan, R., Kuzina, I., Wincovitch, S., Nowotarski, S. H. \& Giniger, E. The Abl/enabled

662 signaling pathway regulates Golgi architecture in Drosophila photoreceptor neurons. Mol.

$663 \quad$ Biol. Cell 25, 2993-3005 (2014).

664 19. Dent, E. W. \& Gertler, F. B. Cytoskeletal dynamics and transport in growth cone motility

665 and axon guidance. Neuron 40, 209-227 (2003). 
666

667

668

669

670

671

672

673

674

675

676

677

678

679

680

681

682

683

684

685

686

687

20. Murray, M. A., Schubiger, M. \& Palka, J. Neuron differentiation and axon growth in the developing wing of Drosophila melanogaster. Dev. Biol. 104, 259-273 (1984).

21. Blair, S. S. \& Palka, J. Axon guidance in cultured wing discs and disc fragments of Drosophila. Dev. Biol. 108, 411-419 (1985).

22. Riedl, J. et al. Lifeact: a versatile marker to visualize F-actin. Nat. Methods 5, 605-607 (2008).

23. Linsmeier, I. et al. Disordered actomyosin networks are sufficient to produce cooperative and telescopic contractility. Nat. Commun. 7, 12615 (2016).

24. Lowery, L. A. \& Vactor, D. V. The trip of the tip: understanding the growth cone machinery. Nat. Rev. Mol. Cell Biol. 10, 332-343 (2009).

25. Dent, E. W. \& Gertler, F. B. Cytoskeletal dynamics and transport in growth cone motility and axon guidance. Neuron 40, 209-227 (2003).

26. Sánchez-Soriano, N. et al. Drosophila growth cones: A genetically tractable platform for the analysis of axonal growth dynamics. Dev. Neurobiol. 70, 58-71 (2010).

27. Lacayo, C. I. et al. Emergence of Large-Scale Cell Morphology and Movement from Local Actin Filament Growth Dynamics. PLoS Biol. 5, (2007).

28. Winkelman, J. D., Bilancia, C. G., Peifer, M. \& Kovar, D. R. Ena/VASP Enabled is a highly processive actin polymerase tailored to self-assemble parallel-bundled F-actin networks with Fascin. Proc. Natl. Acad. Sci. 111, 4121-4126 (2014).

29. Kannan, R. et al. Tyrosine phosphorylation and proteolytic cleavage of Notch are required for non-canonical Notch/Abl signaling in Drosophila axon guidance. Development 145, $\operatorname{dev} 151548$ (2018). 
30. Kuzina, I., Song, J. K. \& Giniger, E. How Notch establishes longitudinal axon connections between successive segments of the Drosophila CNS. Development 138, 1839-1849 (2011).

31. Ennomani, H. et al. Architecture and Connectivity Govern Actin Network Contractility. Curr. Biol. 26, 616-626 (2016).

32. Lenz, M. Geometrical Origins of Contractility in Disordered Actomyosin Networks. Phys. $\operatorname{Rev.X~4,(2014).~}$

33. Lee, H. et al. The microtubule plus end tracking protein Orbit/MAST/CLASP acts downstream of the tyrosine kinase Abl in mediating axon guidance. Neuron 42, 913-926 (2004).

34. Macnab, R. M. \& Koshland, D. E. The gradient-sensing mechanism in bacterial chemotaxis. Proc. Natl. Acad. Sci. U.S. A. 69, 2509-2512 (1972).

35. Wadhams, G. H. \& Armitage, J. P. Making sense of it all: bacterial chemotaxis. Nat. Rev. Mol. Cell Biol. 5, 1024-1037 (2004).

\section{Figure Legends}

\section{Figure 1: Abl regulates TSM1 axon guidance and extension}

Early pupal wing imaginal discs at different developmental stages. A) 10x Micrographs of wing discs immunostained for the neuronal membrane (anti-HRP) and microtubules (mAb22c10). TSM1 and the L1 nerve, as well as GSR and other neurons comprising the developing L3 nerve are highlighted. B-E) 25x micrographs of terminal TSM1 axon phenotypes at $\sim 24 \mathrm{~h} \mathrm{APF.} \mathrm{B)}$ Wildtype TSM1 developmental pattern, (C-E) Examples of observed developmental errors. Yellow arrows in $\mathrm{C}$ and $\mathrm{E}$ highlight aberrant TSM1 axonal phenotypes. The specific phenotypes depicted were not restricted to individual genotypes (C) neur-GAL4; UDicer2; Abl RNAi/Abl4, 
711 (D, E) neur-GAL4; UDicer2; Abl RNAi. F) Histogram plotting the frequency of terminal TSM1

712 developmental errors in the indicated genotypes. $*=\mathrm{P}<0.05, * *=\mathrm{P}<0.01, * * *=\mathrm{P}<0.005$,

$713 * * * *=\mathrm{P}<0.0001 ;$ Fisher's exact T-test.

715 Figure 2: The TSM1 growth cone accumulates actin and is dominated by filopodial

716 protrusions

717 The TSM1 axon was imaged in the intact, explanted wing. Image stacks were segmented and actin

718 intensity was quantified along the axon shaft. (A) 3-dimensional rendering of the cell membrane,

719 and (B) z-projected image of the distal axon of TSM1 from one typical video frame from wild

720 type. In (B), membrane is in red, actin is in green, and the axon shaft has been traced in white. (See

721 supplemental Figure 3 for unmerged color channels and pseudocolored actin channel). C) A fully

722 segmented image of the distal TSM1 axon: protrusions are traced in blue and the axon shaft is

723 traced in white. D) A representative actin distribution extracted from the lifeactGFP signal in the

724 axon in $2 \mathrm{~B}$. The yellow star demarcates the origin of the actin profile. E) A representative

725 distribution of protrusions extracted from the segmented axon in 2B. The yellow star demarcates

726 the origin of the actin profile. F) A 3-dimensional plot of sequential actin distributions extracted

727 over time from a single TSM1 trajectory. Profiles were extracted from images captured every 3

728 minutes. Individual distributions are highlighted to show actin "inchworming". G) Scatter plot of

729 the positions of the protrusion maximum vs the actin maximum along the axon. $\mathrm{n}=338$ timepoints

730 from 14 individual trajectories; Pearson r correlation coefficient; paired t test.

732 Figure 3: Actin leads and predicts the advance of the axonal protrusion density 
733 A) Attributes of a representative actin distribution are annotated to demarcate the length, the

734 maximum and the midpoint of the actin peak, as well as the nominal leading and trailing

735 boundaries of the distribution (the leading and trailing square root of the $2^{\text {nd }}$ moment of the

736 distribution, respectively; see text). B) The maximum of the actin distribution, as well as the

737 leading and trailing 'edges' of the distributions are plotted relative to the position of the maximum

738 protrusion density along the axon, which is set at the origin. Significance of the offset of these

739 actin landmark positions relative to protrusion density in each respective timepoint was determined

740 by two-tailed paired t-test. $\mathrm{n}=338$ timepoints from 14 individual trajectories, $* * * *=\mathrm{p}<0.0001)$

741 Error bars are mean \pm SD. C) A scatter plot of the change in the offset distance between actin and

742 protrusion maxima in a single time-step vs the change in the offset distance in the following time

743 step. $\mathrm{n}=338$ timepoints from 14 individual trajectories; Pearson r. D) Average protrusion length

744 over time is plotted for a $10 \mu \mathrm{m}$ interval centered on the midpoint of the actin distribution at the

745 start of an actin translocation event (red swath), and for the interval centered $20 \mu \mathrm{m}$ distal to that

746 start point (black and white swath). Protrusion length for each $20 \mu \mathrm{m}$ actin midpoint translocation

747 event analyzed was normalized to the summed protrusion length at $\mathrm{t}=0 . \mathrm{n}=128$ time points from

7487 individual trajectories; mean \pm SEM for each time point is plotted over time; Pearson $\mathrm{r}$ correlation

749 coefficient. E) Gallery of images showing a time-course of TSM1 axon extension and actin

750 advance (lifeactGFP, green; and CD4 tandem tomato, red; representative of $\mathrm{n}=14$ independent

751 trajectories). White arrows highlight protrusions fated to disassemble over time as actin advances;

752 white box highlights zone fated to develop dense projections once it is occupied by actin.. See

753 Supplemental Figure 5 for timecourse with unmerged color channels and pseudocolored actin

754 channel). 


\section{Figure 4: Abl perturbed TSM1 axons employ the same cell biology as wildtype}

757 A) Data sets comprising 10 individual parameters extracted from Wildtype, Abl KD and Abl OE

758 TSM1 axons were dimensionally reduced by PCA. Principal components 1 (PC 1) and 2 (PC 2)

759 are plotted for each genotype and are representative of the parameter relationships within all

760 pairwise combinations of the first 4 principal components, which account for $>70 \%$ of the total

761 variance in the data sets (see text Supplemental Figure 6). B) The length of the actin peak, as

762 measured by the sum of the square root of the leading and trailing second moments about the actin

763 peak in each time point (see methods), was quantified in wildtype, $\mathrm{Abl} \mathrm{KD}, \mathrm{ABl} \mathrm{OE}$ and in the

764 Dicer2 actin control. $n>300$ time points from 14 trajectories for each genotype; significance

765 determined by one-way ANOVA; **p $<0.01$, ****p $<0.0001$ n.s. = non-significant. C) The length

766 of the protrusion peak, as measured by the square root of the second moment about the peak in

767 protrusion density in each time point (see methods), was quantified in wildtype, Abl KD and ABl

768 OE. $\mathrm{n}>300$ time points from 14 trajectories for each genotype; significance determined by one-

769 way ANOVA; *p $<0.05, * * * * p<0.0001$. D) Z-scores for actin and morphological parameters

770 were calculated for $\mathrm{Abl} \mathrm{KD}$ (green) and $\mathrm{Abl} \mathrm{OE}$ (red) relative to wildtype.

771

772 Figure 5: Biased stochastic fluctuations of accumulated actin move the distribution forward

773 A) A plot of the axonal positions of landmarks in the actin distribution from a single trajectory

774 over-time. The actin maximum (blue), and the leading (green) and trailing (black) 'edges' of the

775 actin distribution are highlighted. This plot is representative of $n=14$ independent trajectories. B)

776 A histogram of the length of excursions of the actin maximum in single time steps. $\mathrm{n}=324$ time

777 steps from 14 individual wildtype trajectories. C) A scatter plot of the absolute value of the step 
size of the actin maximum versus the length of the actin distribution. $n=324$ time steps from 14 individual wildtype trajectories; Pearson r correlation coefficient.

Figure 6: Abl perturbation fragments the actin distribution and disorganizes its advance

782 A) Fragmentation of the actin distribution, as calculated by the Fisher information content of each

783 actin profile in each time point was quantified in the indicated genotypes. B - D) Representative

784 examples of the mean actin fragmentation phenotype for each genotype. E) The dynamic

785 instability of the actin distribution, as calculated by the Jensen-Shannon Divergence (JSD) of

786 sequential pairs of actin profiles over time (see methods), was quantified in wildtype, Abl KD,

$787 \mathrm{ABl} \mathrm{OE}$ and in the Dicer2 actin control. For $(\mathrm{A})$ and $(\mathrm{E}), \mathrm{n}=338$ time points from 14 trajectories

788 for each genotype; significance determined by one-way ANOVA; ****p $<0.0001$; n.s. = non-

789 significant; error bars indicate standard deviation. $(\mathrm{F}-\mathrm{H})$ The JSD of all pairwise combinations of

790 the actin profiles from all time steps of one individual trajectory from each genotype were

791 calculated, color coded, and are presented as matrices. In each case, the matrix shown represents

792 a trajectory with a mean JSD value that is approximately average for that genotype. (F' $\left.-\mathrm{H}^{\prime}\right)$ 3-D

793 sequential actin distribution plots that correspond to the representative JSD matrices above. I)

794 Scatter plots of the Fisher information versus the length of the protrusion peak for each respective

795 genotype. J) Scatter plot of the absolute value of the actin peak step size versus the length of the

796 actin peak. K) Scatter plot of the Fisher information versus the length of the actin peak. For I - K,

$797 \mathrm{n}>300$ time point from 14 individual trajectories for each genotype; Pearson $\mathrm{r}$ correlation 798 coefficient. 
801 A) Accumulated actin in the distal axon fluctuates in size, transiently invading growth cone

802 protrusions. B) Selective accumulation of actin in a more distal axonal protrusion advances the

803 distribution, and triggers the emergence of nascent protrusions in that region, as well as the

804 disassembly of protrusions in proximal regions no longer bearing enhanced levels of accumulated

805 actin.

806 


\begin{tabular}{|c|}
\hline Measured parameters of the TSM1 growth cone \\
\hline Total number of protrusions \\
\hline Total length of protrusions \\
\hline Average protrusion branching order \\
\hline Growth cone volume \\
(square root of the second moment about the protrusion peak) \\
\hline Length of the actin peak \\
(square root of the second moment about the actin peak) \\
\hline Offset between position of actin maximum vs protrusion maximum \\
\hline Axon extension rate: \\
Rate of change of position of the midpoint of actin peak \\
\hline Fragmentation of the actin distribution: \\
Fisher Information \\
\hline Jensen Shannon Divergence \\
\hline Dynamic instability of the actin distribution: \\
\hline
\end{tabular}


bioRxiv preprint doi: https://doi.org/10.1101/511840; this version posted January 4,2019 . The copyright holder for this preprint (which was not certified by peer review) is the author/funder, who has granted bioRxiv a license to display the preprint in perpetuity. It is made available under aCC-BY 4.0 International license.

Figure 1:

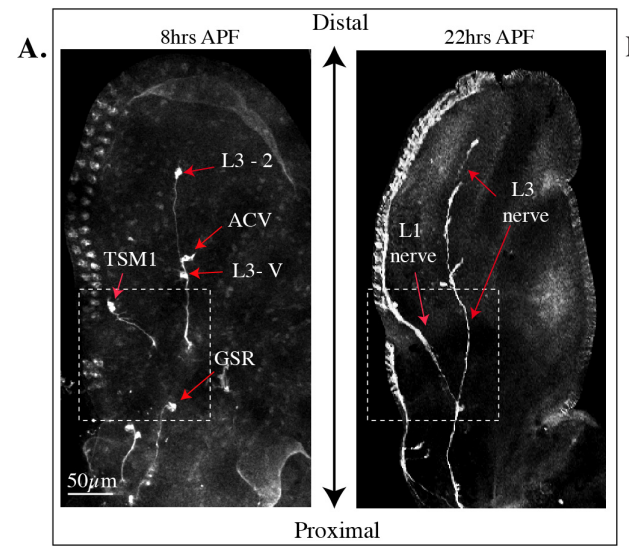

D.

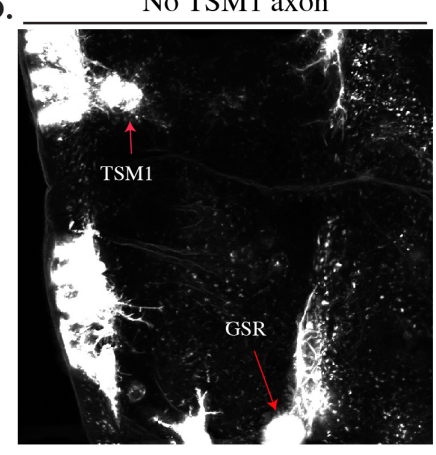

E.

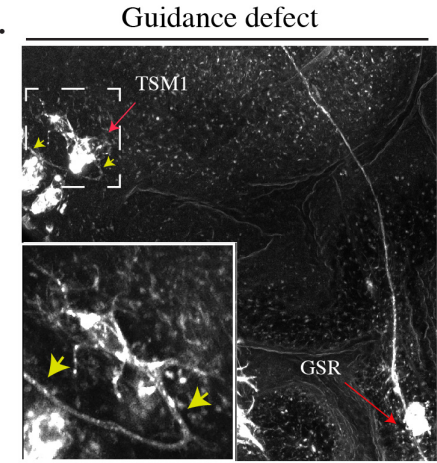

B.

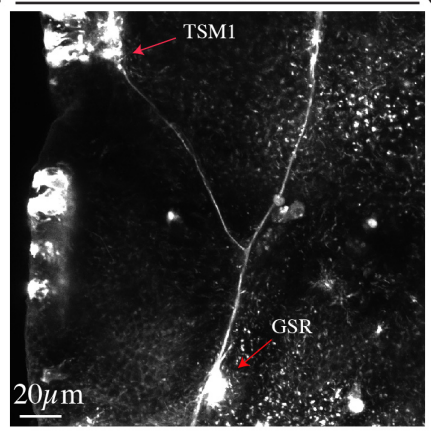

C. Axonal stall w/ ectopic branching
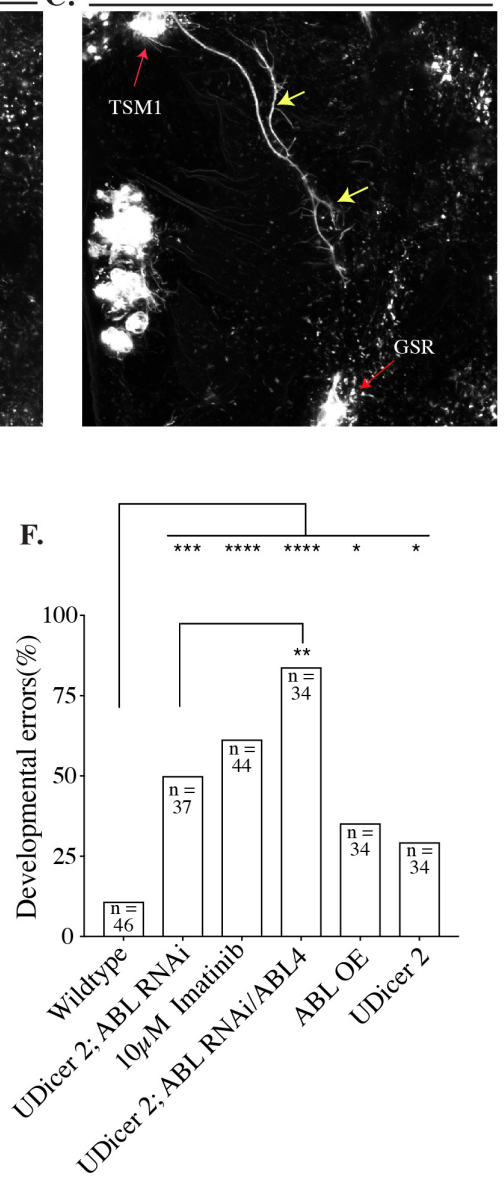
Figure 2

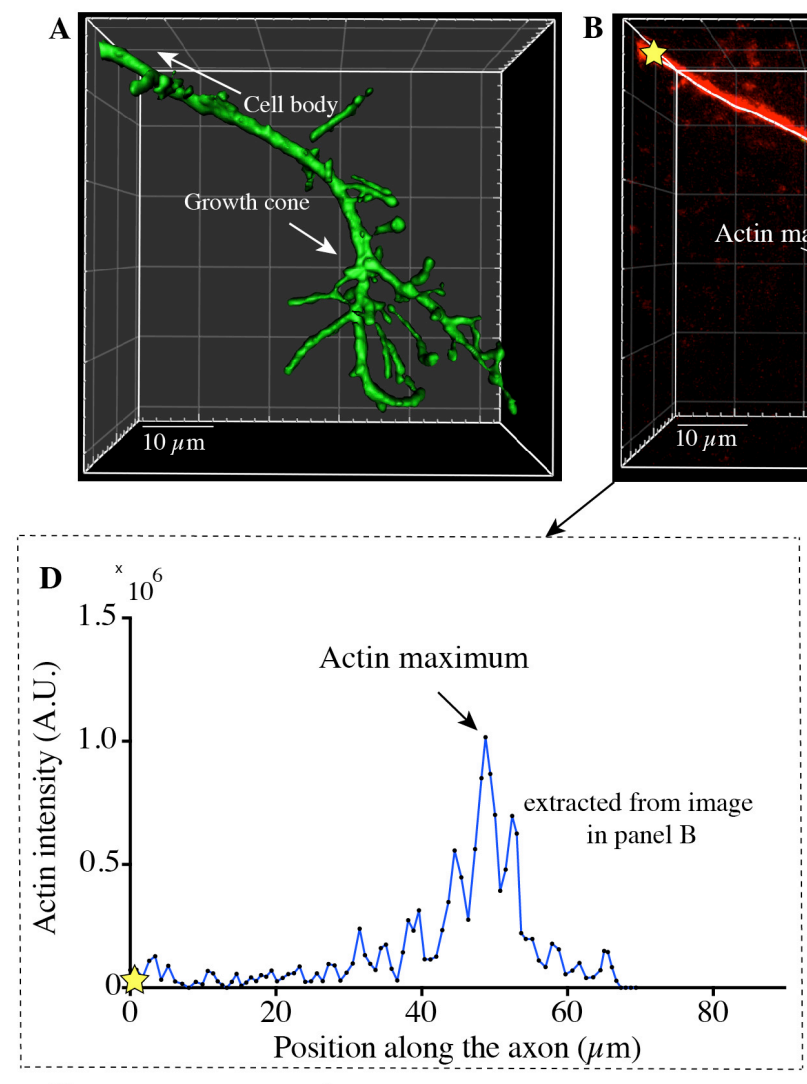

$\mathbf{F}$
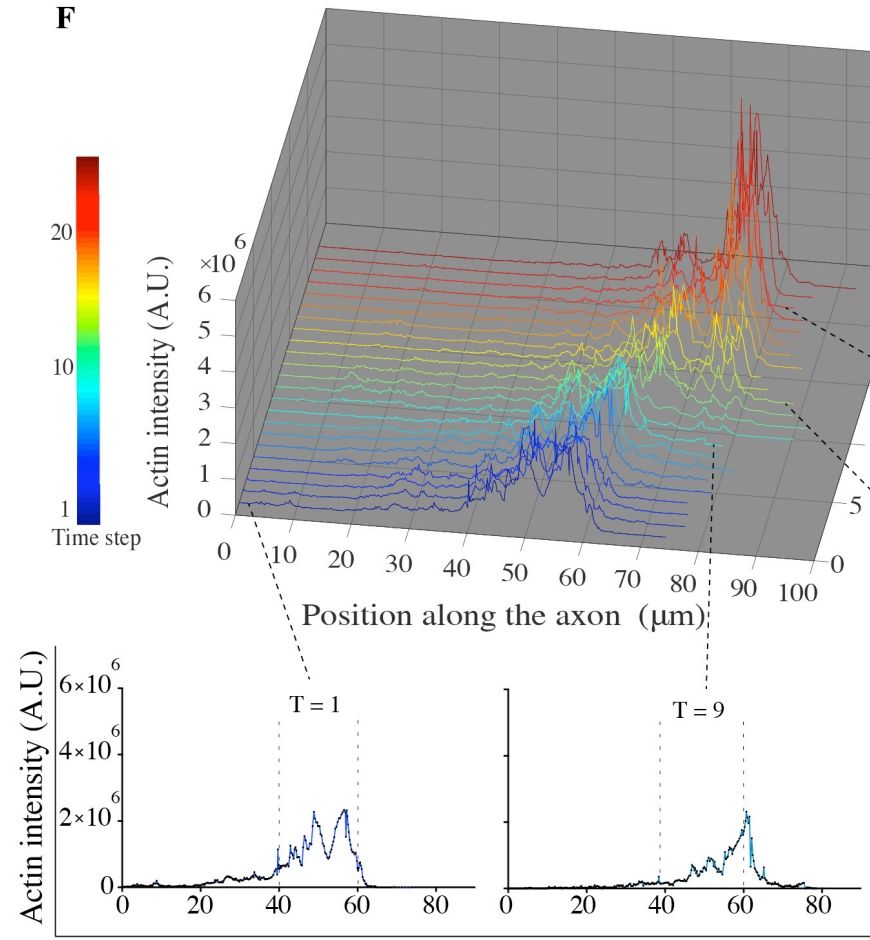
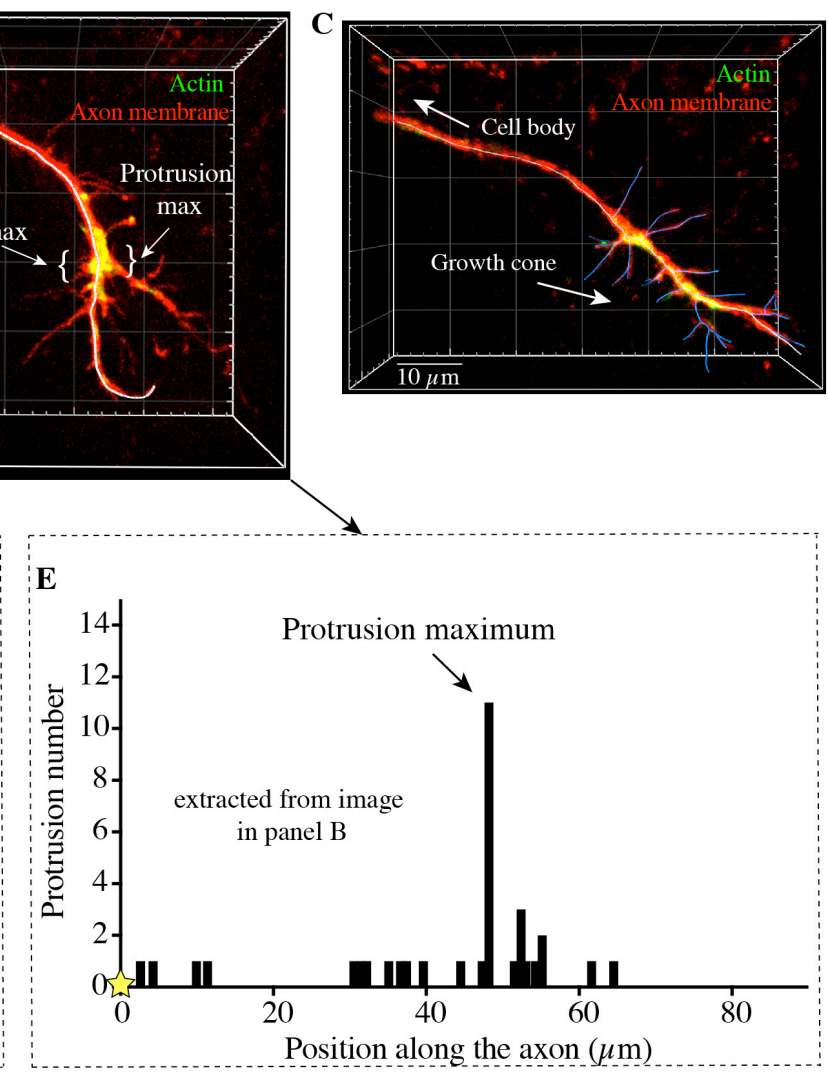

G
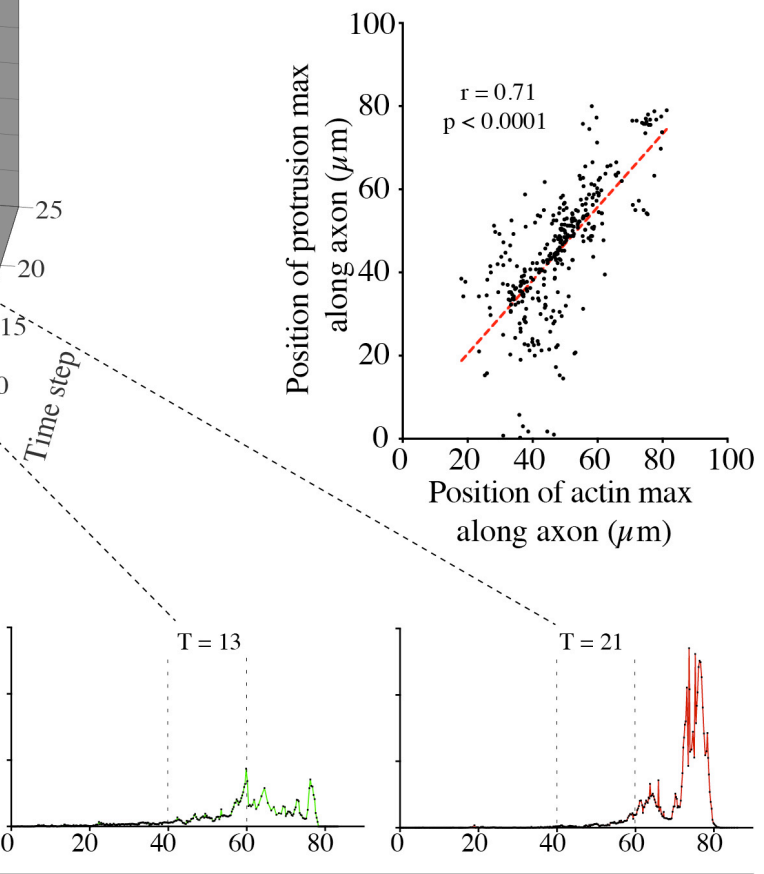

Position along the axon $(\mu \mathrm{m})$ 


\section{Figure 3}

A.

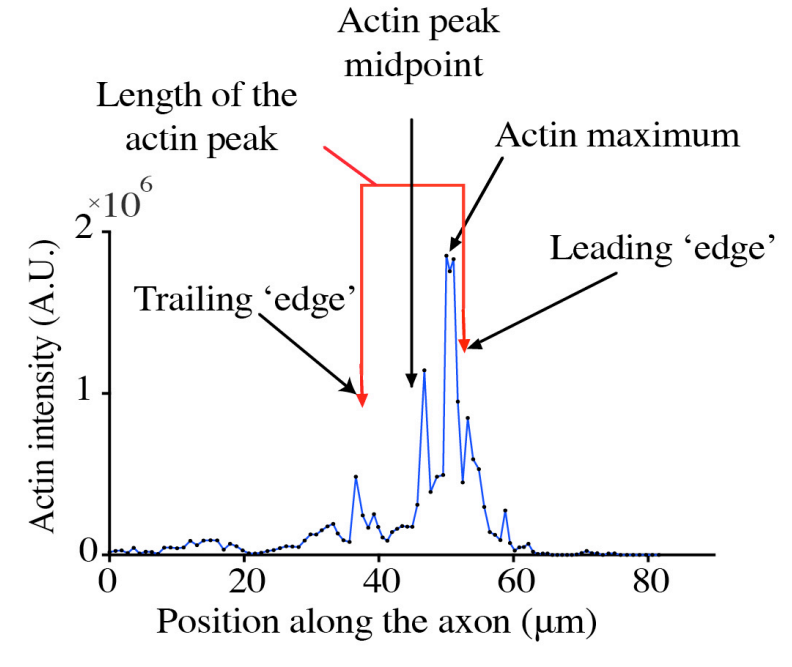

B.

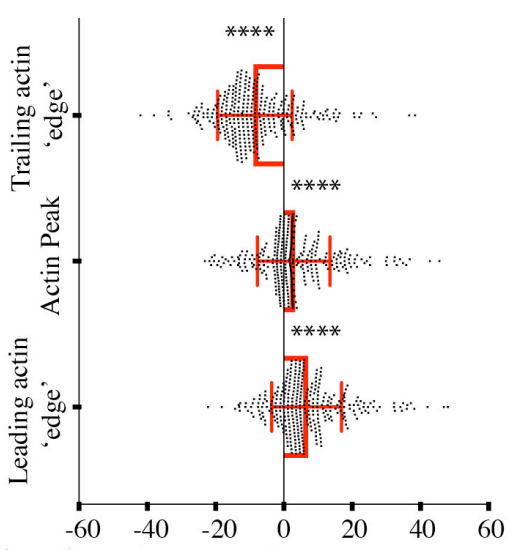

Position along the axon relative to protrusion peak $(\mu \mathrm{m})$
C.

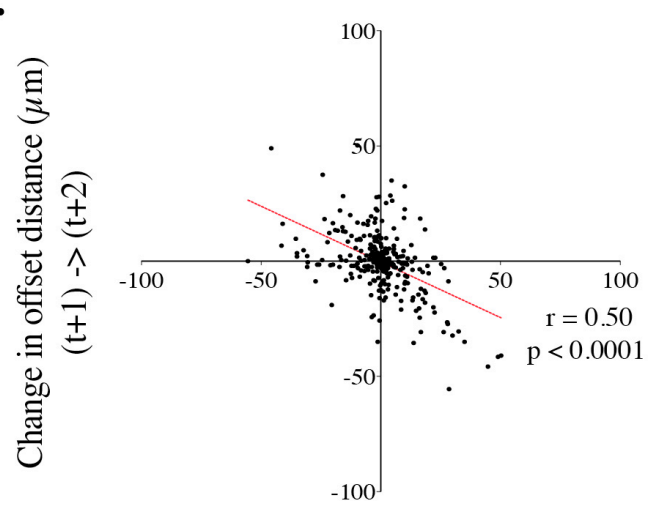

Change in offset distance $(\mu \mathrm{m})$

$(\mathrm{t} 0)->(\mathrm{t}+1)$
D.

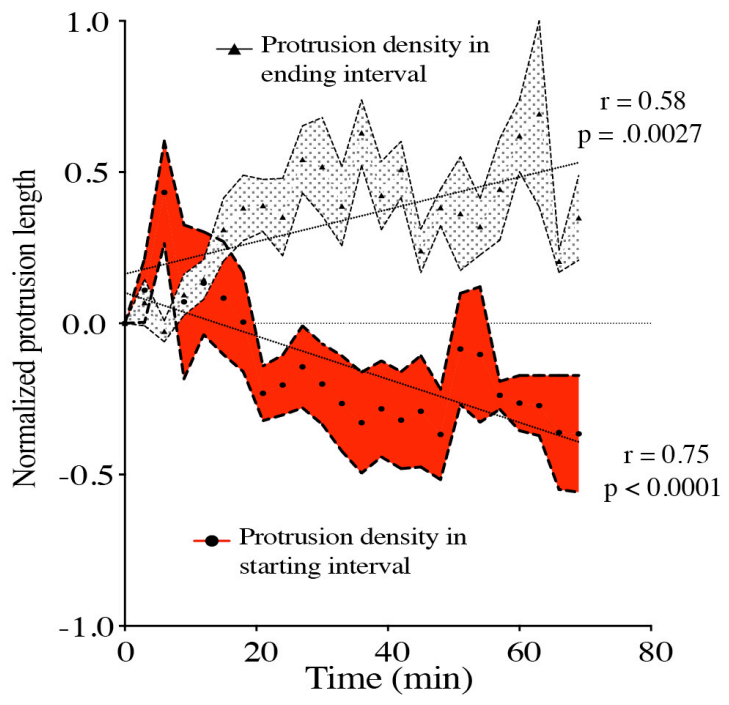

E.
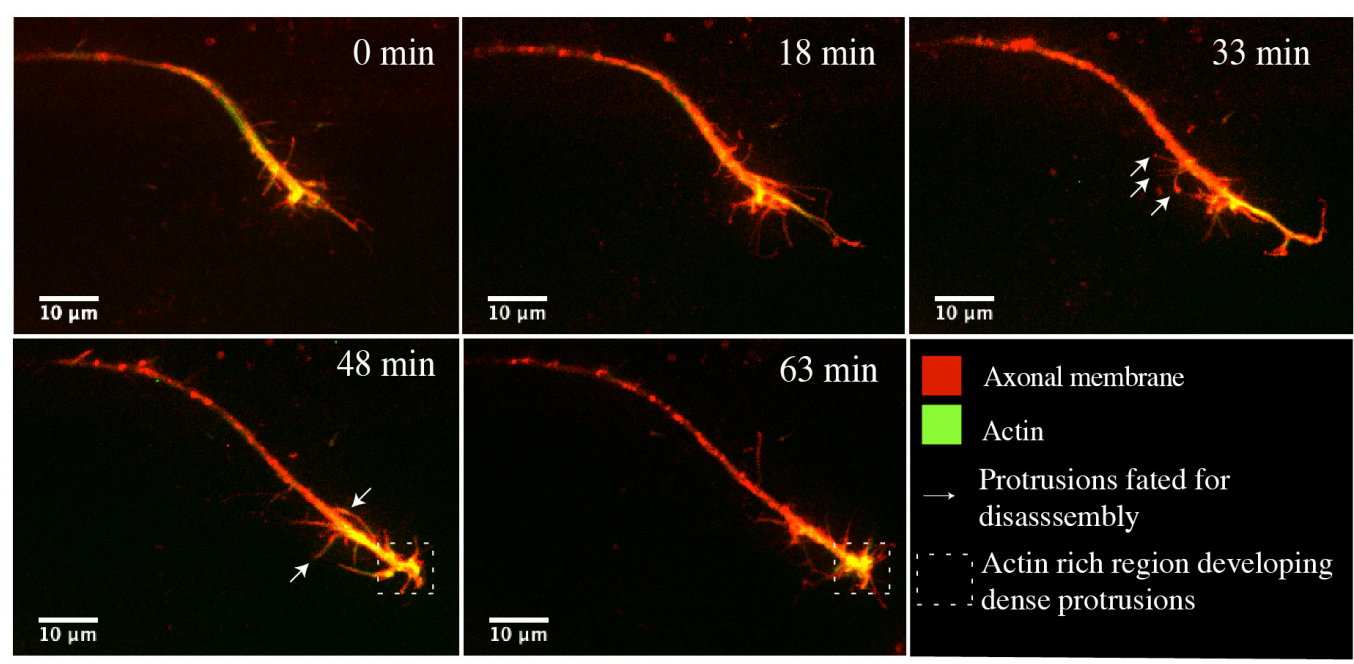
Figure 4
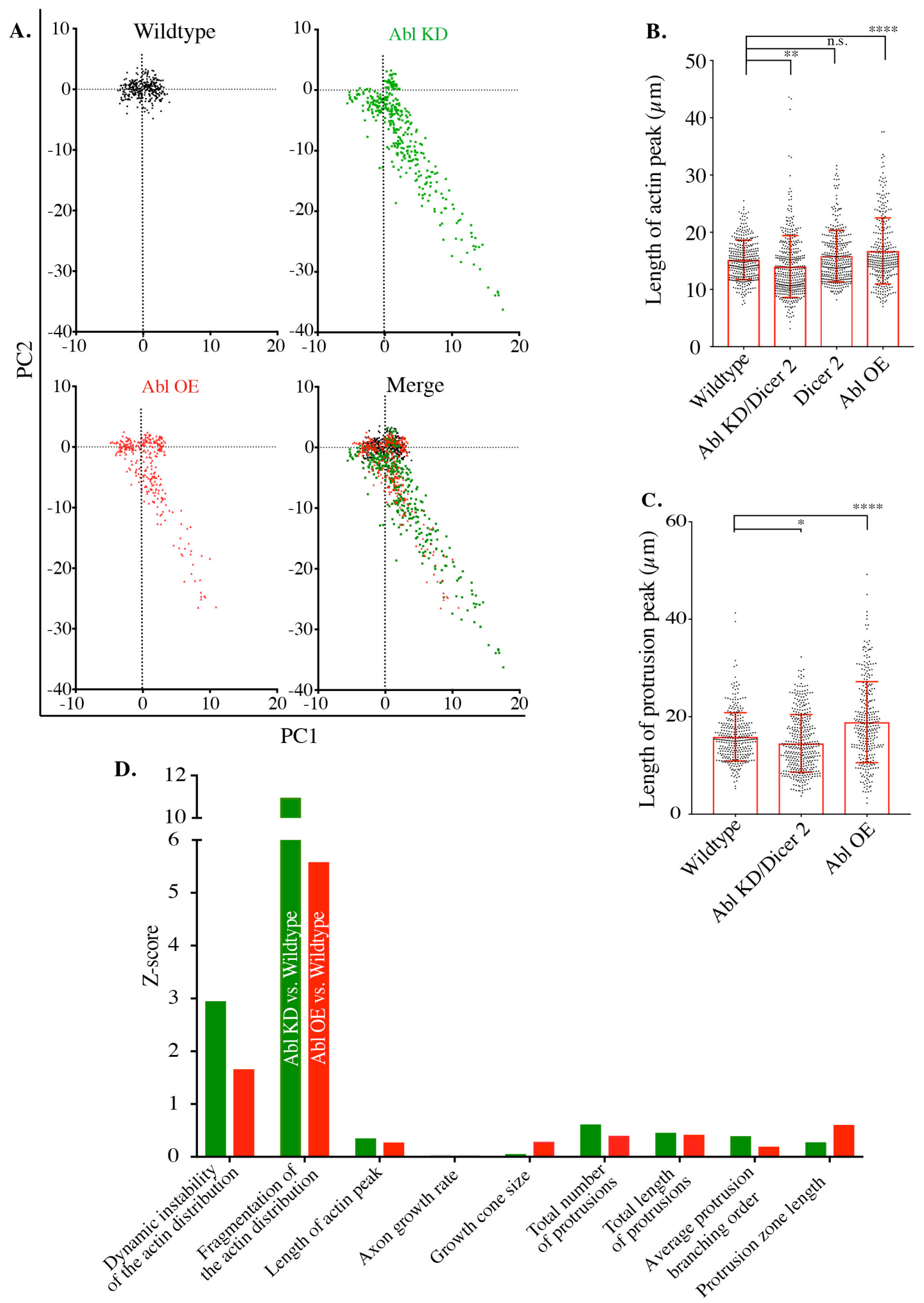
bioRxiv preprint doi: https://doi.org/10.1101/511840; this version posted January 4, 2019. The copyright holder for this preprint (which was not certified by peer review) is the author/funder, who has granted bioRxiv a license to display the preprint in perpetuity. It is made available under aCC-BY 4.0 International license.

Figure 5

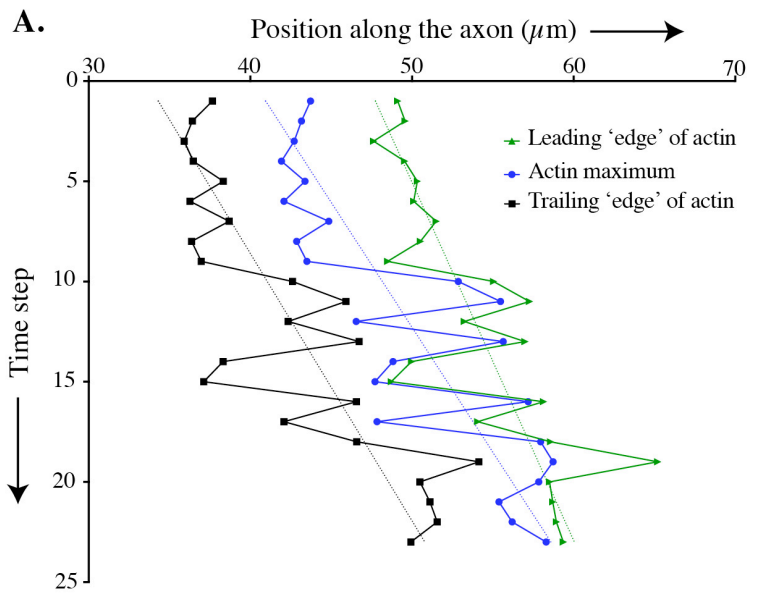

B.

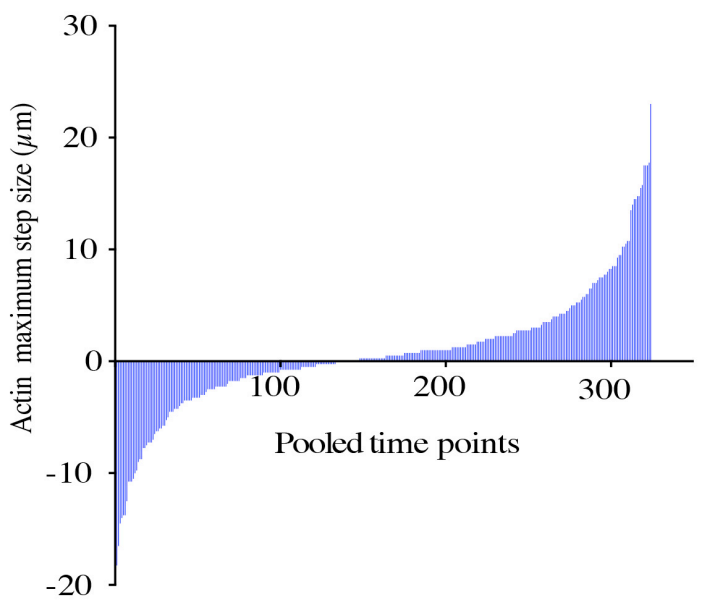

C.

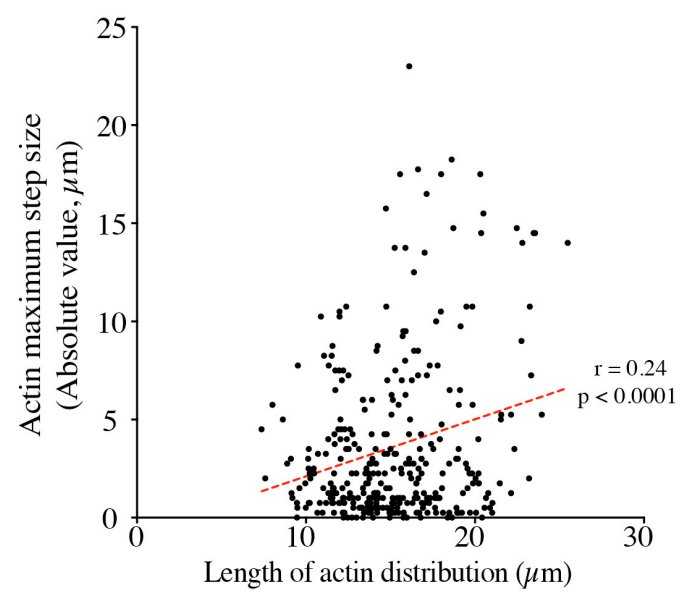


bioRxiv preprint doi: https://doi.org/10.1101/511840; this version posted January 4, 2019. The copyright holder for this preprint (which was not certified by peer review) is the author/funder, who has granted bioRxiv a license to display the preprint in perpetuity. It is made available under aCC-BY 4.0 International license.

A

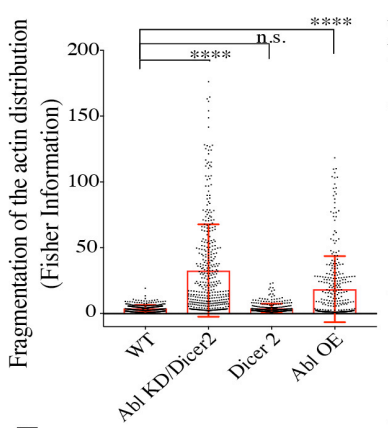

B

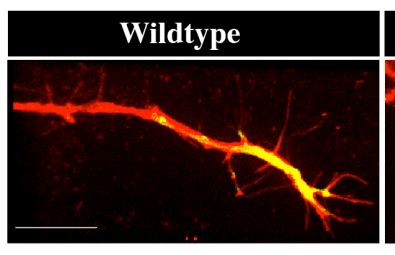

\section{C}

D
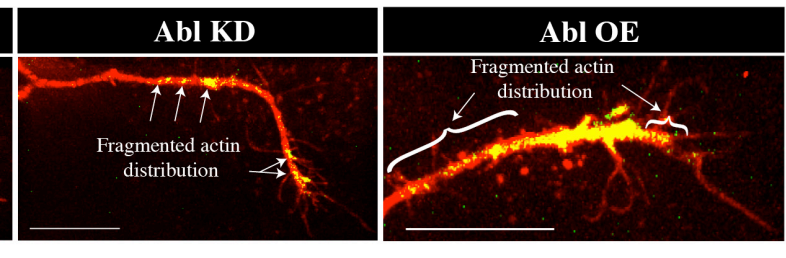

G

$\mathbf{H}$

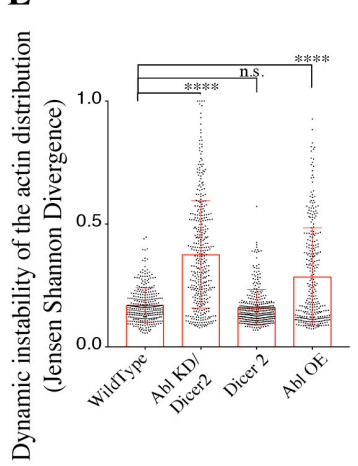

F
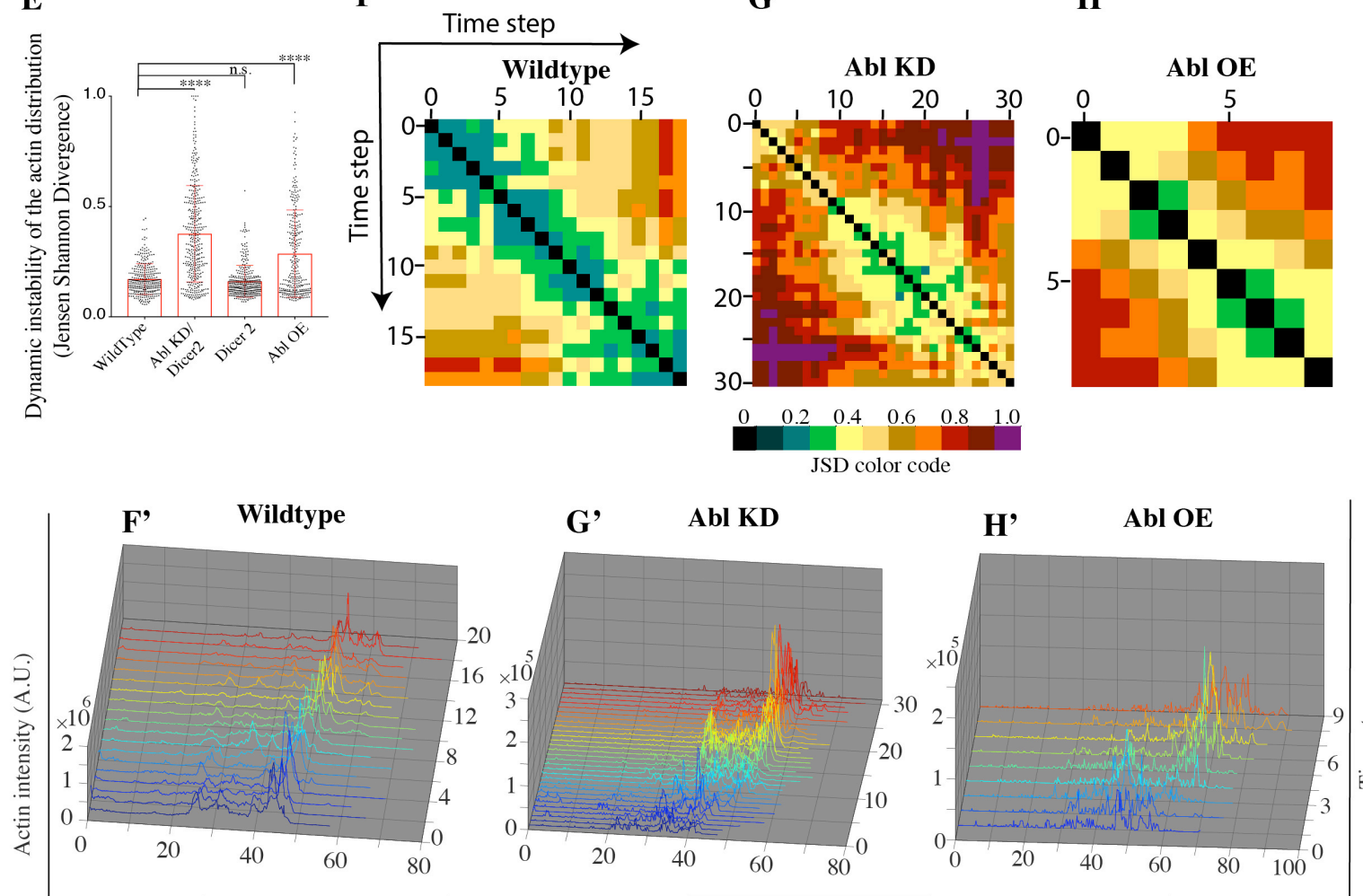

Position along the axon $(\mu \mathrm{m})$

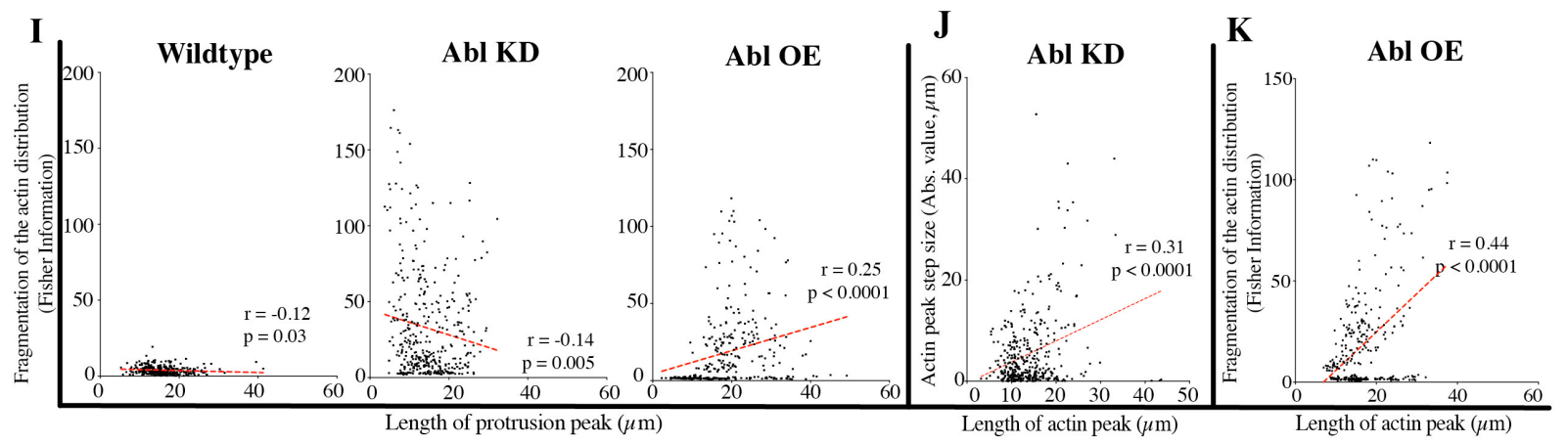


bioRxiv preprint doi: https://doi.org/10.1101/511840; this version posted January 4, 2019. The copyright holder for this preprint (which was not certified by peer review) is the author/funder, who has granted bioRxiv a license to display the preprint in perpetuity. It is made available under aCC-BY 4.0 International license.

\section{Figure 7}

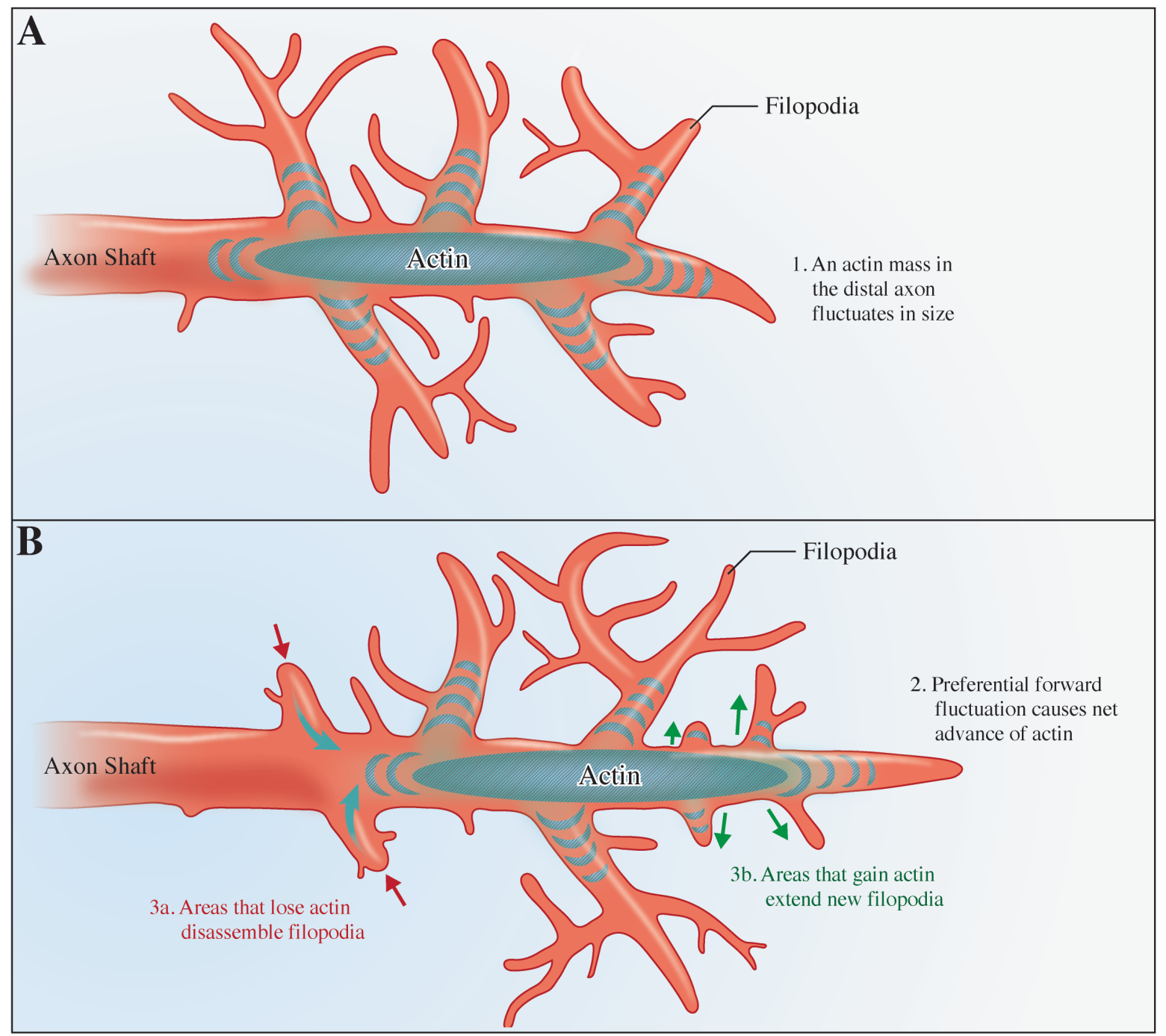


bioRxiv preprint doi: https://doi org/10 1101/511840; this version posted January 4, 2019. The copyright holder for this preprint (which was not certified by peer review) is the author/funder, who has granted bioRxiv a license to display the preprint in perpetuity. It is made available under aCC-BY 4.0 International license.

\section{Supplemental Figure 1}

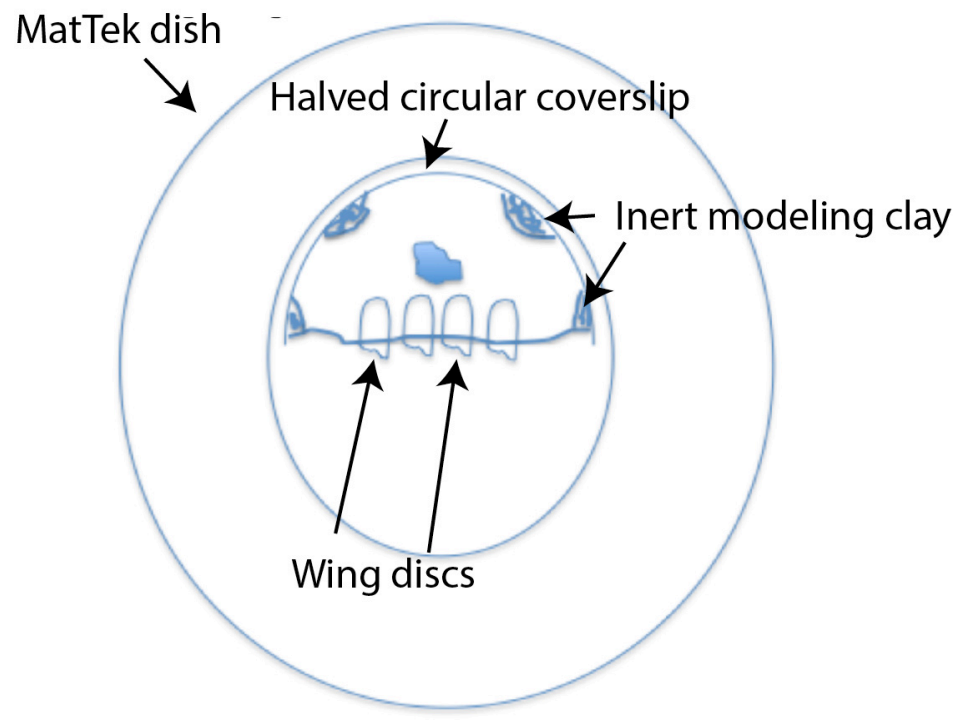

836 Supplemental Figure 1: Mount schematic for culturing and imaging TSM1 

aCC-BY 4.0 International license.

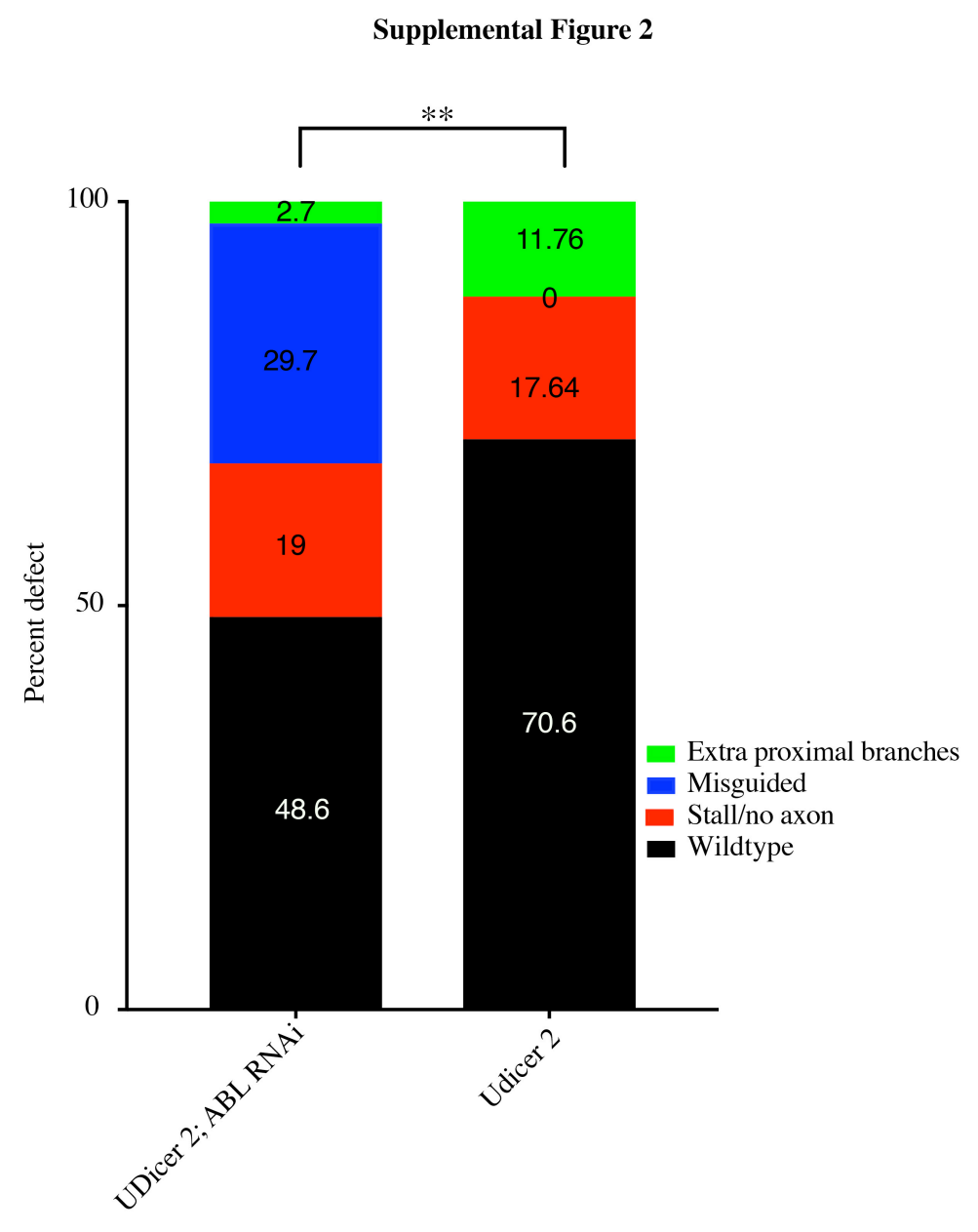

840 Supplemental Figure 2: Abl KD enhanced by Dicer2 causes a different spectrum of defects

841 than that caused by Dicer 2 alone. Chi-square; ** $\mathrm{p}=0.0035$.

842

843

844 
Supplemental Figure 3:

850 Supplemental Figure 3: Actin accumulates at the site of the TSM1 growth cone

851 A) A max intensity projection of the membrane localized tandem tomato signal is presented

852 alongside B), a summation projection of the lifeactGFP signal and C), a merge of both channels.

853 The lifeactGFP summation projection is pseudocolored in D) to detail local GFP intensity values.

854 Fiji/ImageJ was used for all image processing in this gallery. 


\section{Supplemental figure 4}

A

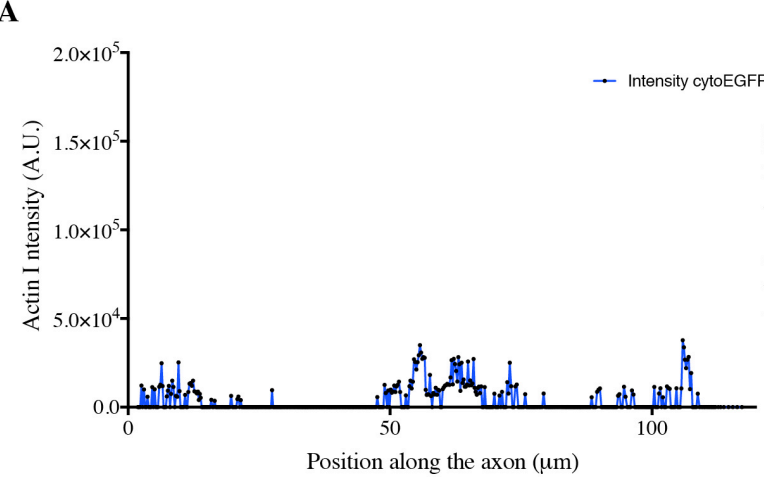

B

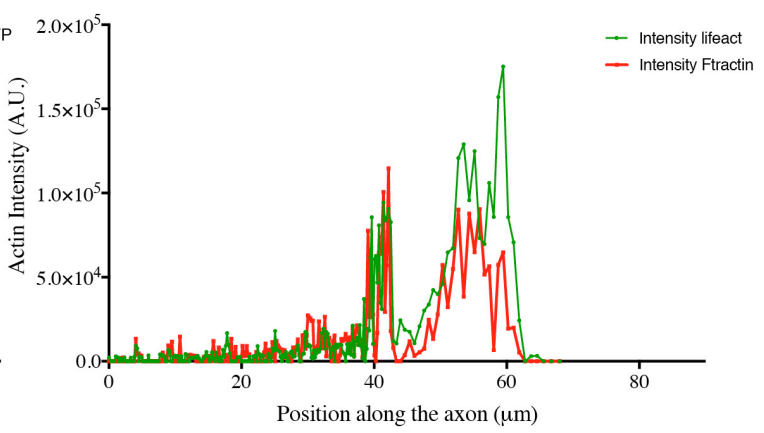

864 Supplemental Figure 4: Different actin markers, but not cytoplasmic eGFP, accumulate in a

\section{5 distal mass in the TSM1 axon}

866 A) A representative actin distribution extracted from a TSM1 axon expressing cytoplasmic eGFP.

867 B) An overlay of two actin distributions from a single TSM1 axon expressing both the lifeactGFP

868 and F-tractin transgenes. Each plot is representative of $n=5$ independent trajectories, with $>15$

869 time points per trajectory. 


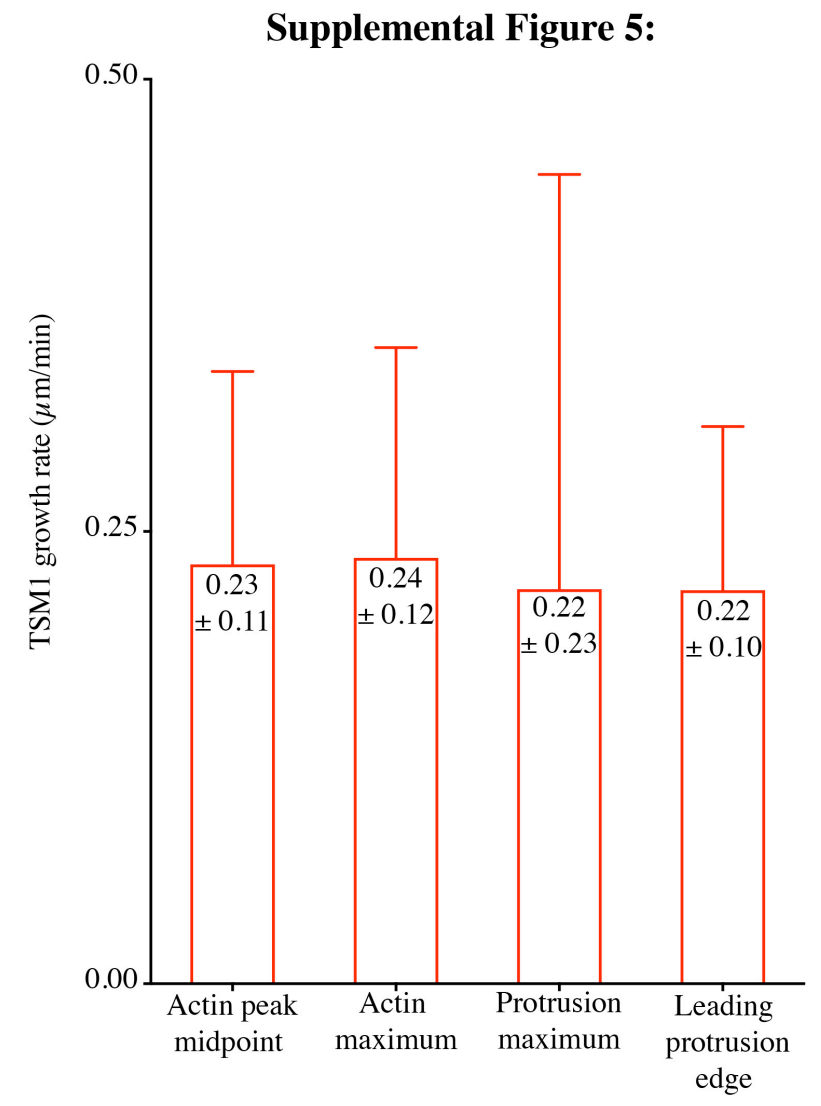

\section{Supplemental Figure 5: TSM1 growth rate}

873 The indicated features of the TSM1 growth cone were tracked independently over time. Mean \pm

874 SEM are reported. Measures of growth rate are not significantly different as analyzed by ANOVA

875 and paired T-test.

876 


\section{Supplemental Figure 6}

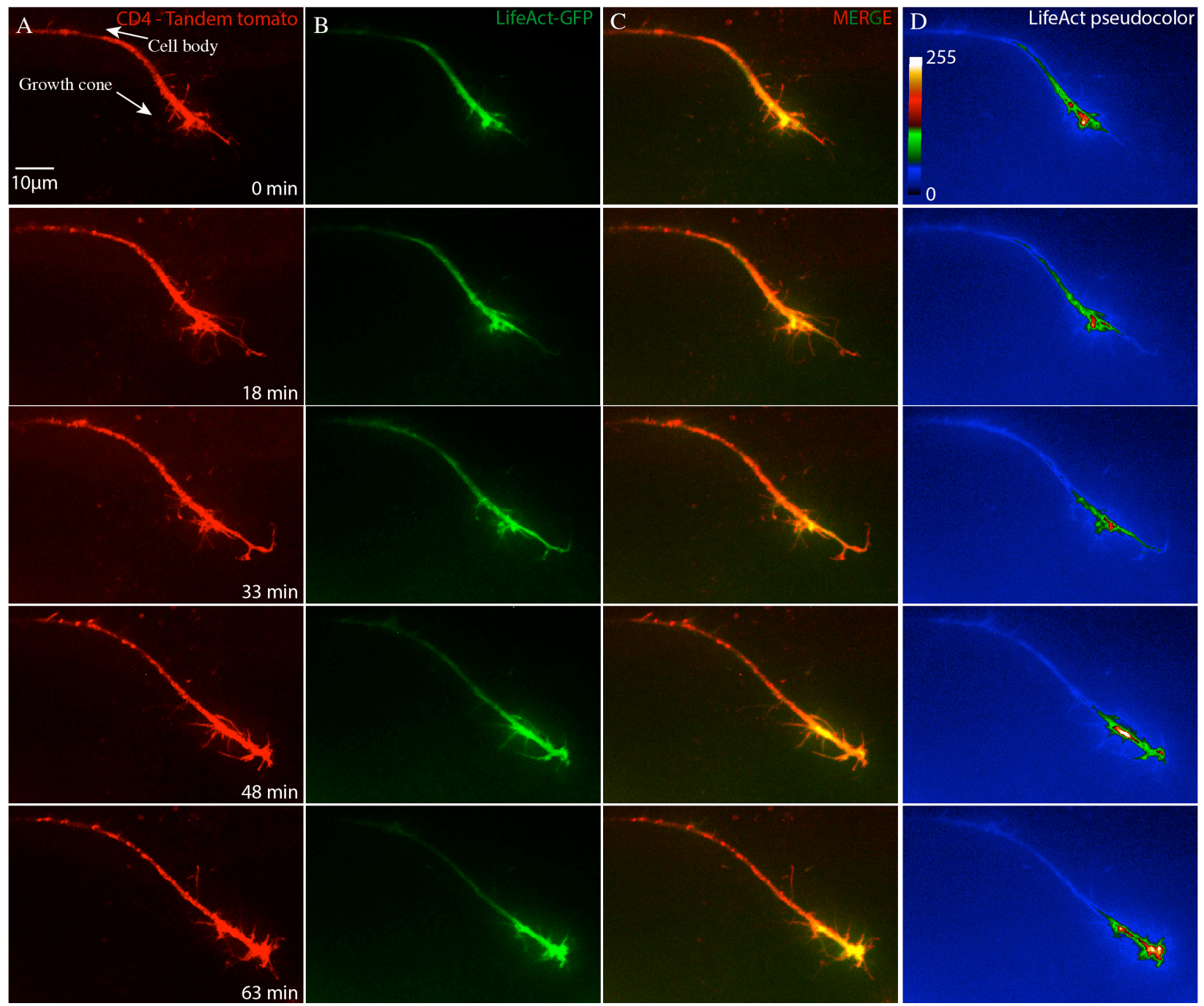

\section{Supplemental Figure 6: Actin accumulation advances the TSM1 growth cone}

879 A time course gallery of (A) a max intensity projection of the membrane localized tandem tomato

880 signal, (B) a summation projection of the lifeactGFP signal, (C) a merge of the membrane and

881 actin channels, and (D), A pseudocolored lifeact summation projection detailing local GFP

882 intensity values. Fiji/ImageJ was used for all image processing in this gallery. 


\section{Supplemental Figure 7}

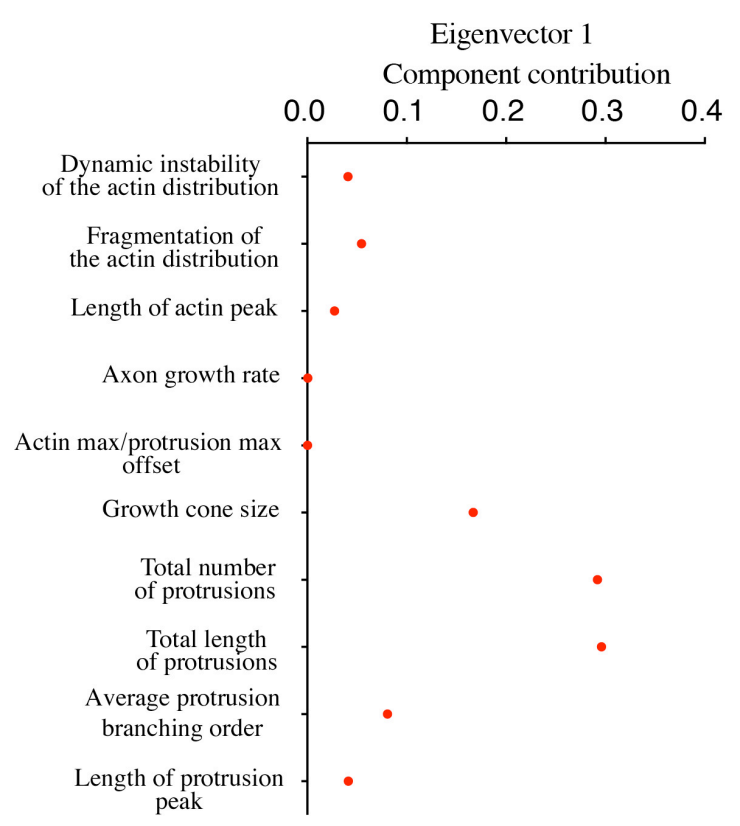

Eigenvector 3

Component contribution

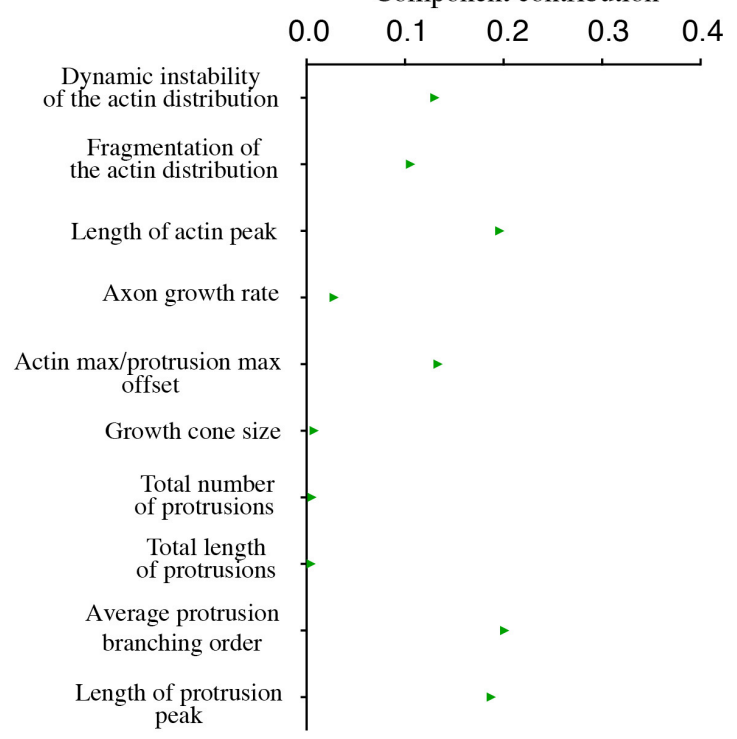

Eigenvector 2

Component contribution

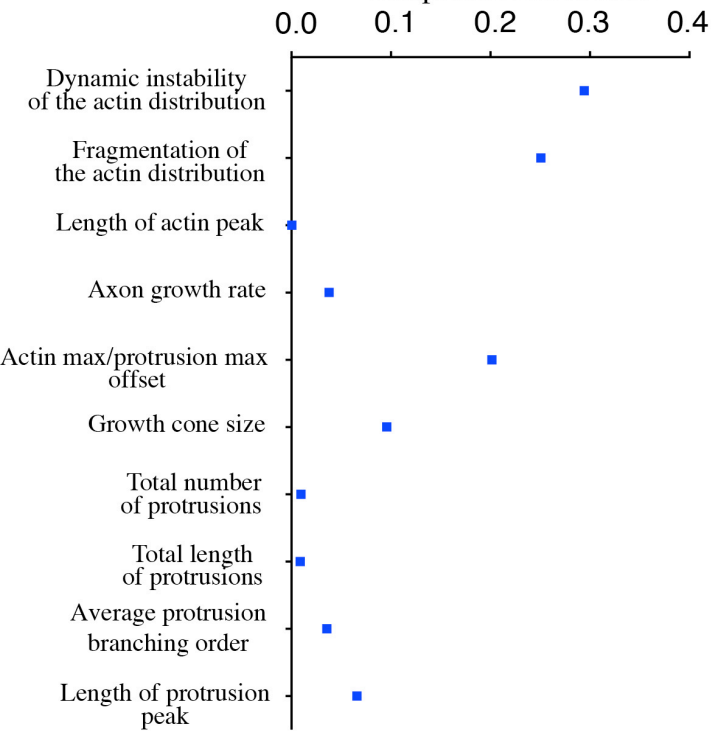

Eigenvector 4

Component contribution

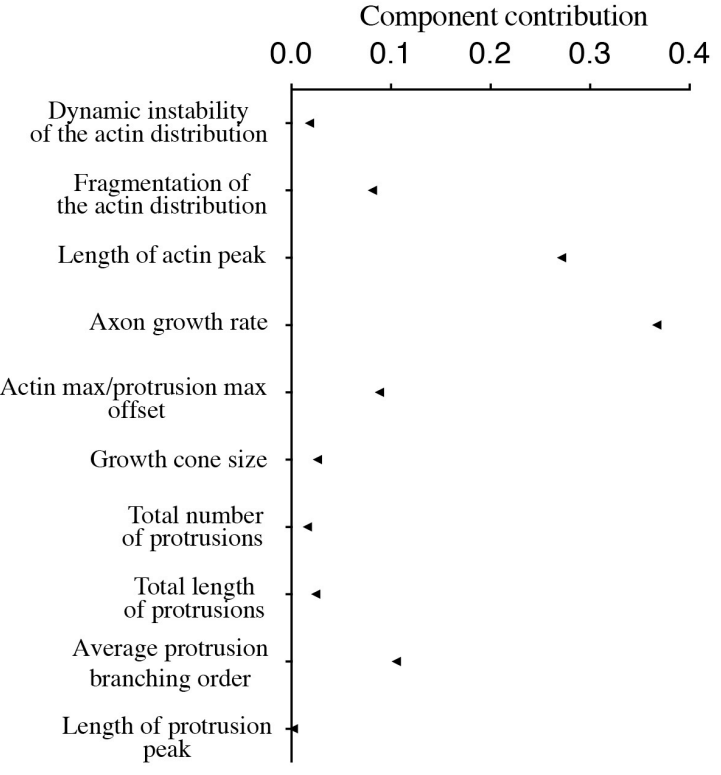

\section{Supplemental Figure 7: Parameter contributions to the first four principal components}

887 Principal component analysis was performed using 10 measured variables that quantify aspects of

888 TSM1 axonal growth. The first four eigenvectors account for $\sim 71 \%$ of the total variance in the

889 data set. 


\section{Supplemental Figure 8}

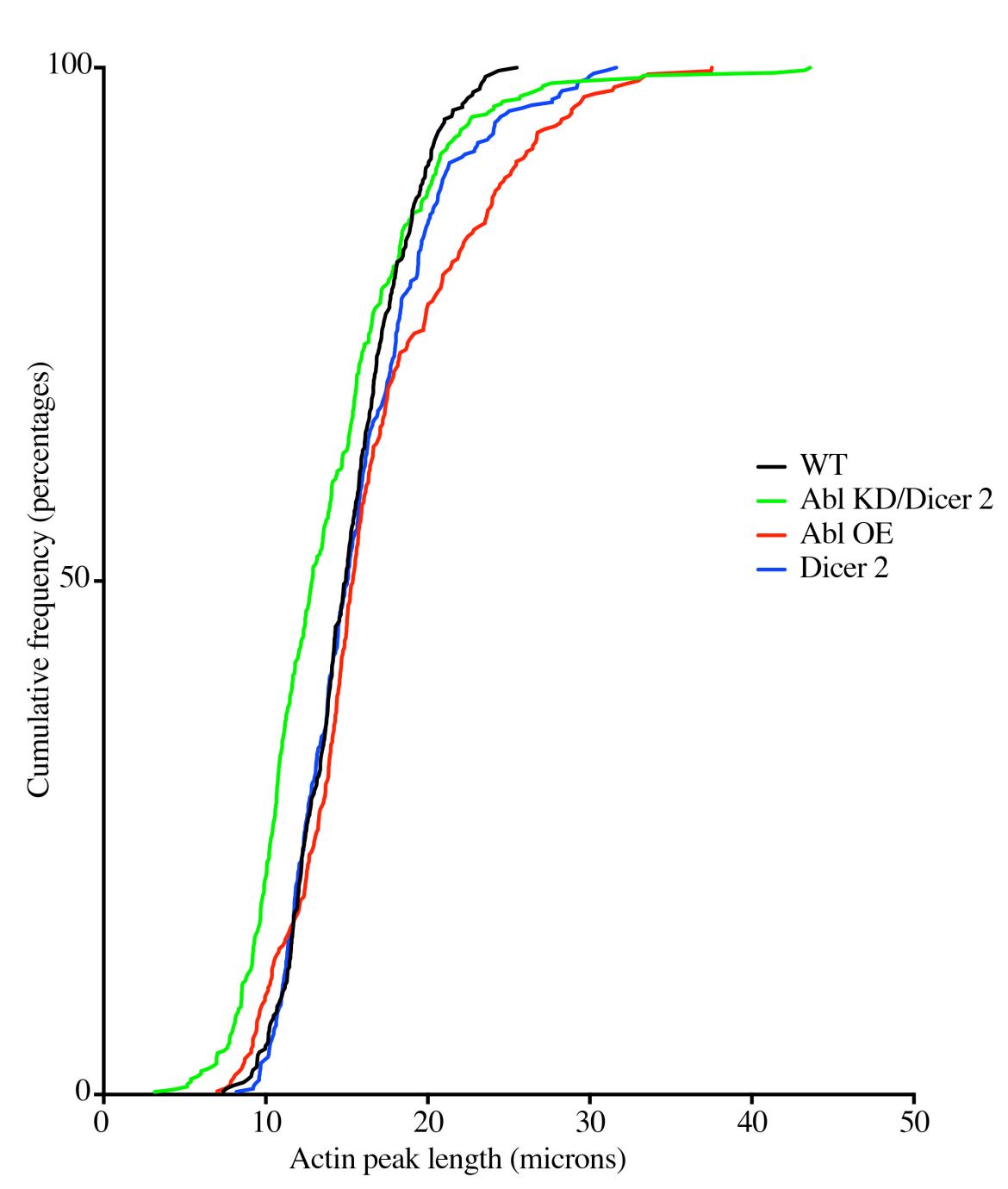

\section{Supplemental Figure 8: Cumulative frequency of actin distribution sizes}

893 The lengths of the actin peak for each genotype are presented as cumulative frequency distributions

894 to visualize the contribution of actin peaks of different lengths to each data set. 